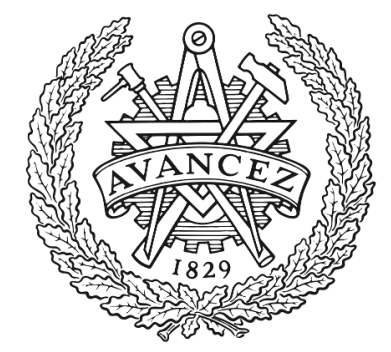

\title{
CHALMERS
}

UNIVERSITY OF TECHNOLOGY

\section{Verification of the Random Line-of-Sight Measurement Setup at 1.5-3 GHz including MIMO Throughput Measurements of a Complete Vehicle}

Downloaded from: https://research.chalmers.se, 2023-04-26 14:41 UTC

Citation for the original published paper (version of record):

Schilliger Kildal, M., Mansouri Moghaddam, S., Razavi, A. et al (2020). Verification of the Random Line-of-Sight Measurement Setup at 1.5-3 GHz including MIMO

Throughput Measurements of a Complete Vehicle. IEEE Transactions on Vehicular Technology, 69(11): 13165-13179. http://dx.doi.org/10.1109/TVT.2020.3021250

N.B. When citing this work, cite the original published paper.

(O2020 IEEE. Personal use of this material is permitted.

However, permission to reprint/republish this material for advertising or promotional purposes 


\title{
Verification of the Random Line-of-Sight Measurement Setup at 1.5-3 GHz Including MIMO Throughput Measurements of a Complete Vehicle
}

\author{
Madeleine Schilliger Kildal, Sadegh Mansouri Moghaddam, Aidin Razavi, Jan Carlsson, Senior Member, IEEE, \\ Jian Yang, Senior Member, IEEE, and Andrés Alayón Glazunov, Senior Member, IEEE
}

\begin{abstract}
The performance evaluation of wireless systems is crucial for the development of future systems with more connected devices. It is essential to have an easy and relevant method for ensuring the wireless communication performance of the devices. We have recently developed a new random lineof-sight (random-LOS) measurement system for evaluating the communication performance of wireless devices, e.g., transceivers and antennas installed on a vehicle. In the measurement system, a plane wave is generated in the test zone emulating the far-field wave transmitted from a radio base station. In this paper we present both numerical simulations and actual experimental results of the random-LOS over-the-air (OTA) measurement setup operating in the $1.5-3 \mathrm{GHz}$ band. The measurement accuracy is determined by the field variations within the test zone, where a smaller variation gives better measurement accuracy. In this paper the achieved accuracy expressed in terms of standard deviation (STD) was evaluated to be approximately $1 \mathrm{~dB}$ of the power within a cylindrical test zone of height $0.4 \mathrm{~m}$ and diameter $2 \mathrm{~m}$. The active multiple-input multiple-output (MIMO) performance of antenna systems installed on an actual vehicle was measured and evaluated using the presented setup. A comparison to a theoretical zero forcing $(\mathrm{ZF})$ receiver is also presented.
\end{abstract}

Index Terms-OTA, vehicular communication, reflector, random-LOS, throughput.

\section{INTRODUCTION}

W E are moving towards a future with more connected devices including also those mounted on vehicles. With the introduction of the fifth generation of wireless

Copyright (c) 2015 IEEE. Personal use of this material is permitted. However, permission to use this material for any other purposes must be obtained from the IEEE by sending a request to pubs-permissions@ieee.org.

M. S. Kildal is both with the Department of Electrical Engineering, Chalmers University of Technology, Gothenburg 412 96, Sweden and RanLOS AB, Gothenburg 436 34, Sweden (e-mail: madeleine.kildal@ chalmers.se).

S. M. Moghaddam is with the Department of Electrical Engineering, Chalmers University of Technology, Gothenburg 412 96, Sweden (e-mail: sadegh.mansouri@chalmers.se).

A. Razavi is with Ericsson Research, Ericsson AB, Gothenburg, Sweden (e-mail: aidin.razavi@ericsson.com)

J. Carlsson is with Chalmers University of Technology, RanLOS $\mathrm{AB}$, as well as Provinn $\mathrm{AB}$, Gothenburg 411 05, Sweden (e-mail: jan.carlsson@provinn.se)

Jian Yang is with the Department of Electrical Engineering, Chalmers University of Technology, Gothenburg 412 96, Sweden (e-mail: jian.yang@chalmers.se)

A. A. Glazunov is with the Department of Electrical Engineering, University of Twente, P.O. Box 217, 7500 AE Enschede, The Netherlands and he is also affiliated with the Department of Electrical Engineering (E2), Chalmers University of Technology, Gothenburg, Sweden (e-mails: a.alayonglazunov@utwente.nl, andres.glazunov@chalmers.se).

Manuscript received Month Date, Year; revised Month Date, Year.? communication $(5 \mathrm{G})$ and self-driving vehicles, the wireless communication performance of these devices will play a crucial role. Therefore, ensuring that the required communication performance is actually realized has become more relevant than ever before. One way to do this is to test the wireless communication performance of the installed devices by using over-the-air (OTA) test methods.

There exist several different methods for OTA testing focusing on active system-level performance evaluation. Most of them were primarily targeting testing of smaller devices, such as mobile phones, laptops, etc. They have been developed for the current 2G, 3G, $4 \mathrm{G}$ frequencies below $6 \mathrm{GHz}$, and can be categorized in three main techniques: the multiprobe anechoic chamber (MPAC) [1], [2]; the reverberation chamber (RC) [3], [4]; and the radiated two-stage method [5].

The introduction of devices with larger form-factor, such as vehicles, require adjustments of these testing methods. An initial investigation of the MPAC for vehicular testing at the $5.9 \mathrm{GHz}$ band, has shown promising results [6]. The RC has been shown to be useful for testing devices with form factor larger than $42 \mathrm{~cm}$ [7]. A version of the radiated two-stage method, the wireless cable, has also shown potential for being able to be used for evaluating antenna systems on vehicles [8]. However, there is still no standardized method for active system evaluation of vehicular mounted antennas.

A useful hypothesis for OTA characterization techniques has been formulated in [9]. In the hypothesis, two edge propagation environments are introduced: the random line-ofsight (random-LOS) and the rich isotropic multipath (RIMP) environments. These edge environments are linked through the formulation of the hypothesis, "If a wireless device is tested with good performance in both pure-LOS and RIMP environments, it will also perform well in real-life environments and situations, in a statistical sense".

The RIMP environment is a well-understood environment, commonly emulated in an RC for evaluation of devices. The $\mathrm{RC}$ makes use of many scatterers resulting in a large number of waves impinging at the antennas of the device under test (DUT) [10]. Various studies have been done on the RC OTA evaluation of system performance in multipath channels, and many useful results have been generated [11]-[13].

The second edge environment, the random-LOS, is the one considered in this paper, which can be realized in a traditional anechoic chamber (AC) or semi-anechoic chambers. The emulated environment is a type of pure line-of-sight 


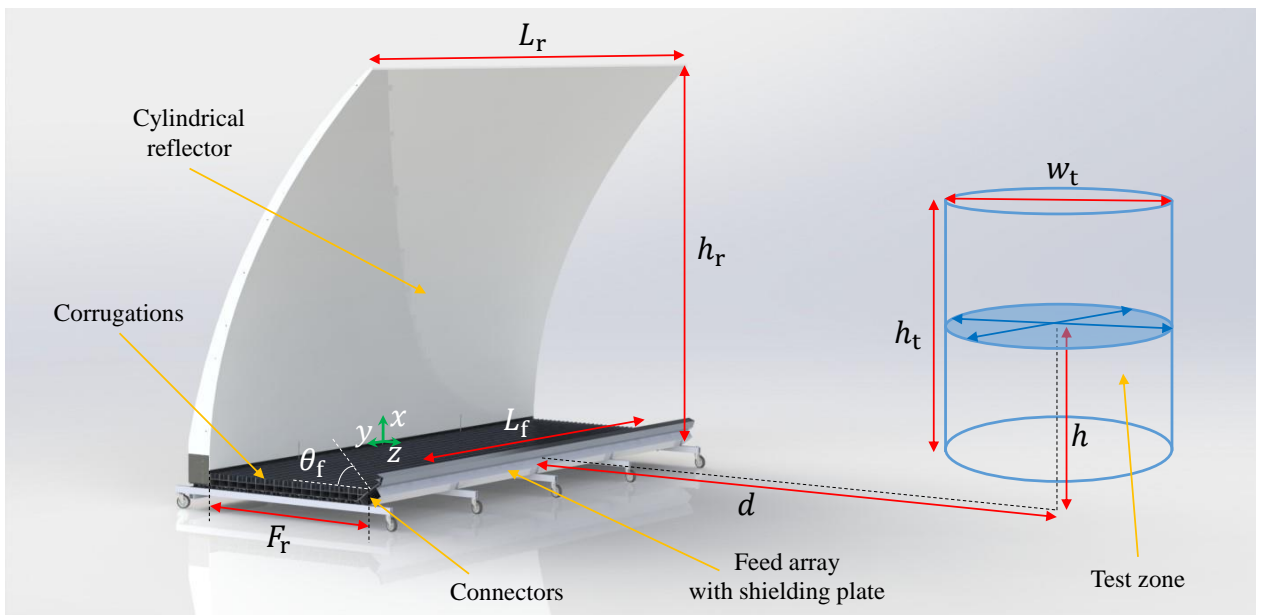

Fig. 1. Drawing of the cylindrical reflector with the dual-polarized linear array feed of bowtie antennas and the cylindrical test zone in front.

(LOS) environment, i.e., a free-space channel. The randomness comes into account through the assumption that the angle of arrival (AoA) and the polarization of the impinging waves are random variables. The impinging waves from one AoA are assumed to be at most two, with independent amplitude and phase of orthogonal polarization. This randomness comes from the random orientation and location of the devices. Traditional pure LOS channels have both the transmitter and the receiver fixed, which will result in a fixed angle-of-arrival/departure and polarization. The randomness introduced by the usage of mobile devices comes from the movements and the orientation changes, which induce random AoA and polarization of the impinging waves [9]. The random-LOS environment can be exampled by an open highway type of environment for cars, where there is a large distance between the scatterers and a strong dominant signal, i.e., a LOS signal, that comes from the base station. The user devices are operating in the farfield of base station antennas, which means that the performance evaluations also should be performed in the far-field. There are different ways to generate the desired plane wave characteristics of the far-field, for example with a reflector antenna or a planar array [14]-[16]. How well the plane wave characteristics are met determines the accuracy of the system, where a performance closer to the ideal plane wave will give a lower measurement uncertainty. In this paper the field variations will be expressed in terms of standard deviation (STD) of the normalized power and phase within the test zone.

The random-LOS environment has previously been introduced in [17]-[23]. The focus of these papers has been on introducing the random-LOS environment concept and evaluating different realization methods for the test environment. The investigated frequency band in these papers has been from $750 \mathrm{MHz}-6 \mathrm{GHz}$ for automotive applications. In [23], simulations and measurements of a virtual planar array antenna as a random-LOS system have been evaluated. Since a planar array solution becomes expensive to manufacture, simulations of a more cost-effective solution consisting of a cylindrical reflector design was therefore presented in [22]. The proposed reflector solution presented in [22] is scalable to different frequency bands, with applications relevant to other smaller or larger devices. This paper builds upon and extends the work presented in [22]. The investigation has been performed for the frequency range $1.5 \mathrm{GHz}-3 \mathrm{GHz}$ to demonstrate the method's applicability, covering various long term evolution (LTE) frequency bands. However, the system is scalable to other frequencies, by exchanging the feed array, e.g., $3 \mathrm{GHz}-6 \mathrm{GHz}$, which covers the $802.11 \mathrm{p}$ vehicular communication standard.

The random-LOS reflector setup is similar to a compact antenna test range (CATR) [24]. However, the random-LOS setup is employing a parabolic cylindrical reflector, whereas it is more common with paraboloidal reflectors with a point feed in the CATR. The CATR are usually high precision test ranges with typically a peak-to-peak phase variation of $\pm 5^{\circ}$ and a peak-to-peak amplitude variation of $\pm 0.5 \mathrm{~dB}$ within the quiet zone [25]. The random-LOS setup has a higher field variation, but is instead aiming towards a more cost-effective solution for easy use in the development phase of antennas and wireless devices. The setup is flexible and can be temporarily placed in semi-AC and simpler outdoor open area test sites.

The main contributions of this paper are: 1) the development of the reflector-antenna-based random-LOS OTA test setup at the $1.5 \mathrm{GHz}-3 \mathrm{GHz}$ band, with performance comparisons of the system in terms of simulations and measurements; 2) investigation of the undesired field variations in the test zone, which can be reduced by means of amplitude tapering of the feed array; 3) radiation pattern measurements of a vehicle roof-mounted antenna, including the full vehicle, using the random-LOS measurement setup; and 4) active $2 \times 2$ multipleinput multiple-output (MIMO) measurements performed for the first time on an actual vehicle using the measurement setup.

The remainder of the paper is organized as follows. A general introduction to the random-LOS measurement setup is provided in Section II, where the reflector antenna system is described in detail, including the realization of the reflector. The evaluation methodology of the system in terms of numerical and experimental characterization is presented in Section III. The results of the numerical and experimental evaluation together with analysis are presented in Section IV. 


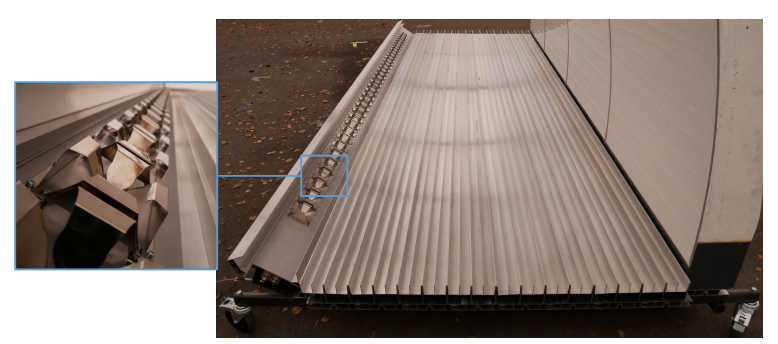

Fig. 2. The feed array of 32 dual-polarized bowtie antennas, plus two extra elements on each side, mounted on the base of metal corrugations in front of the cylindrical reflector antenna.

Radiation pattern measurement results performed on a roofmounted antenna including a full vehicle are presented in Section V. The active $2 \times 2$ MIMO antenna measurements including a full vehicle are presented in Section VI. Conclusions are given in Section VII.

\section{RANDOM-LOS OTA MEASUREMENT SETUP}

The random-LOS OTA measurement setup consists of a cylindrical reflector illuminated by a feed array and metal corrugations as shown in Fig. 1. The overall outer dimensions of the whole setup are $4.2 \mathrm{~m} \times 1.8 \mathrm{~m} \times 3.3 \mathrm{~m}$ (width $\times$ dept $\times$ height). The whole structure is mounted on wheels, such that it can be easily moved in and out of the measurement tent/chamber.

\section{A. Feed Array}

The uniform linear feed array consists of 32 dual-polarized bowtie antenna elements, plus two additional dummy elements on each side, see Fig. 2. The dummy elements are used to provide similar embedded element pattern for all elements of the array. The feed array is a larger version of the 8-element bowtie array described in [26]. The operating frequency of the array is from $1.5 \mathrm{GHz}$ to $3 \mathrm{GHz}$.

The array element is a dual-polarized self-grounded bowtie antenna with a balun. The bowtie antenna element has a slightly different realization from the one described in [26]. In the current realization, the inter-element spacing has been reduced to $10 \mathrm{~cm}$. The smaller spacing was achieved by flipping two of the four "petal feet" inwards towards the center of each element. The petals located with the side towards the neighboring elements in the array were the one affected by the design change. This change was done in order to increase the working frequency of the bowtie array and avoid grating lobes at the higher frequencies.

The elements in the array are excited with uniform phase and amplitude. This is done by using a distribution network made of power splitters and cables. For each polarization, the array elements are combined in groups of 8 elements to four $8 \times 1$ power splitters (Mini-Circuits ZB8PD-362-S+, $600-3600 \mathrm{MHz}$ ). The outputs of these four power splitters are combined in a $4 \times 1$ power splitter (Mini-Circuits ZN4PD1$63 \mathrm{HP}-\mathrm{S}+, 250-6000 \mathrm{MHz})$. The output of this last power splitter is connected to the output connector of the whole feed array. For both polarizations this gives a total number of eight $8 \times 1$ and two $4 \times 1$ power splitters.

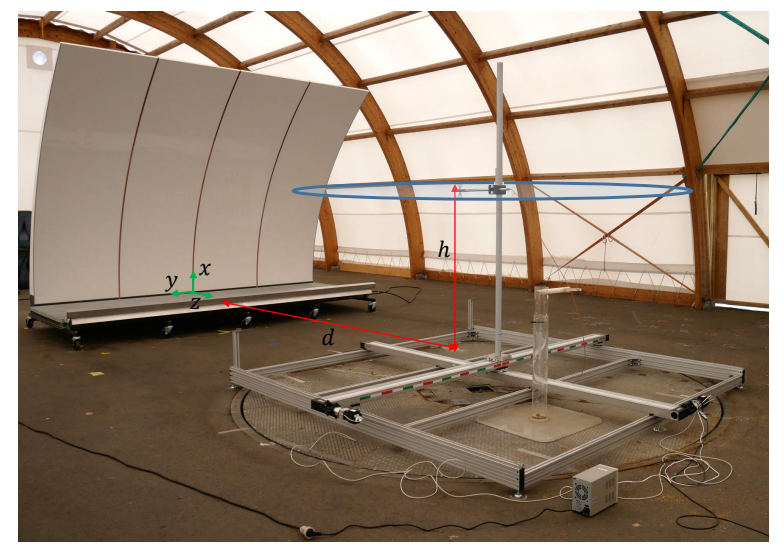

Fig. 3. The random-LOS measurement setup and positioning grid with the biconical probe antenna.

The 32 bowtie elements ( +4 dummy elements) are all mounted on a $4 \mathrm{~m}$ long metal rail that is attached to the corrugation profiles at the base of the reflector as shown in Fig. 2. The feed array has a length of $L_{f}=3.2 \mathrm{~m}$ excluding the dummy elements. There is a shielding plate covering the back side of the feed array, where the baluns and the power splitters are mounted together with all the cables, see Fig. 1.

\section{B. Reflector}

The shape of the cylindrical paraboloidal reflector antenna is given by $z(x, y)=x^{2} /\left(4 F_{r}\right)$, where the focal depth of the reflector is $F_{r}=1.5 \mathrm{~m}$. The reflector has the length $L_{r}=4.2 \mathrm{~m}$ and the height $h_{r}=3 \mathrm{~m}$, see Fig. 1. The shape has been obtained by molding fiberglass together with an aluminum (Aluminum alloy 6082) metal sheet into the shape of the reflector, see Fig. 3. A thin protective coating is covering the reflector. The reflector is made of four molded pieces that have been mounted together side-by-side, by using screws on the back side. The reflector structure is modular, hence the width can be extended. The seams between the four pieces are covered with a copper foil tape (3M 1181) with a conductive adhesive and a width of $2.5 \mathrm{~cm}$.

\section{Assembly Details}

The four reflector pieces are mounted on a metal base structure for stabilization. On top of this base structure, metal corrugations are placed to remove the effect of the ground plane between the feed array and the reflector. The corrugations have a depth of $43 \mathrm{~mm}$ and a width of $40 \mathrm{~mm}$, and are designed to work for the operating frequency band of the system. The corrugations are made of extruded aluminum profiles. They cover the whole base between the reflector and the feed array, with an area of $1.5 \mathrm{~m} \times 4.2 \mathrm{~m}$. The corrugations are divided into smaller parts in a group of three corrugation profiles, and full length of $4.2 \mathrm{~m}$. These groups are then placed next to each other to fill the space between the reflector and the feed array. Two additional corrugations are mounted on the other side of the feed array (not just between the feed array and the reflector) as shown in Fig. 2. These two additional corrugations reduce the direct radiation from the feed array 
into the test zone. For each array element, they also provide the same beamwidth in the transverse plane for both polarizations throughout the band. The feed array is mounted, such that the phase center of the bowtie elements is located in the focal line of the reflector, tilted towards the reflector with an angle of $\theta_{f}=55^{\circ}$.

The whole system can operate at other frequencies than the ones investigated in this paper, by changing the feed array. The feed array can be easily dismounted from the metal structure and can in the future be exchanged for a feed array operating at for example $750 \mathrm{MHz}-1.5 \mathrm{GHz}$ or $3 \mathrm{GHz}-6 \mathrm{GHz}$ to cover other sub- $6 \mathrm{GHz}$ frequency bands. The corrugations are also easily exchangeable for different frequency bands. The whole structure has a weight of around $450-500 \mathrm{~kg}$, where each reflector piece weights around $70-80 \mathrm{~kg}$. The feed array antenna together with the reflector antenna, will hereafter be called the measurement antenna.

\section{FIELD CHARACTERIZATION}

\section{A. Numerical Characterization}

The numerical characterization of the system has been performed using an in-house physical optics (PO) code implemented in Matlab. The full description of the PO code can be found in [22]. The feed sources in the PO code are given by the embedded far-field radiation pattern of the bowtie elements in the feed array. The embedded radiation patterns of the bowtie elements were simulated using CST microwave studio software. The simulations of the embedded radiation pattern include two corrugations on the edge of the feed array (as in the manufactured version) as well as $1 \mathrm{~m}$ of corrugations, corresponding to the corrugations between the feed array and the reflector. However, the corrugations were otherwise not included in the PO code.

In the PO code the incident magnetic field $\mathbf{H}^{\mathrm{i}}$ on the reflector surface was obtained from the embedded radiation pattern of the bowtie elements in the linear feed array. The reflector surface was divided in a grid of square cells, with each having a width not larger than $\lambda / 2$. The PO current $\mathbf{J}=2 \hat{\mathbf{n}} \times \mathbf{H}^{\mathrm{i}}$ was computed from $\mathbf{H}^{\mathrm{i}}$ at the center of each cell. The scattered field $\mathbf{E}^{\mathrm{S}}$ was then calculated from the PO current using the formulas in [10, Sec. 4.2]. The total field $\mathbf{E}^{\mathrm{t}}$ in the test zone was then calculated from the sum of the scattered field $\mathbf{E}^{\mathrm{S}}$ and the incident field $\mathbf{E}^{\mathrm{i}}$ from the bowtie elements. The simulations were performed over $1.5-3 \mathrm{GHz}$.

To obtain the field variations within the test zone in front of the reflector, the total field $\mathbf{E}^{\mathrm{t}}$ was simulated in a grid volume covering $x \in[0 \mathrm{~m}, 3 \mathrm{~m}], y \in[-2 \mathrm{~m}, 2 \mathrm{~m}]$ and $z \in[4 \mathrm{~m}, 7 \mathrm{~m}]$. The grid spacing $d_{x, y, z}$, was the same in all directions with $d_{x}=d_{y}=d_{z}=5 \mathrm{~cm}$, which corresponds to $\lambda / 2$ at $3 \mathrm{GHz}$. For different figures in the result, different parts of these simulated data were extracted. The origin of the coordinate system is at the center of the base of the reflector, as shown in Fig. 1. The total number of grid points in the simulated volume was 400221.

The phase center of the self-grounded bowtie elements in the feed array is located in the focal line of the reflector, corresponding to a height equal to the base of the reflector.
The array structure will block radiation rays from the bottom part of the reflector, since the feed elements are tilted with $\theta_{f}=55^{\circ}$, see Fig. 1 . In reality the bottom $0.1 \mathrm{~m}$ of the reflector will be blocked by the feed array. However, this is not part of the PO-computations, and therefore not accounted for in the simulations.

\section{B. Experimental Characterization}

The experimental characterization setup of a manufactured random-LOS measurement system is described next. The measurement antenna was placed inside a large tent in an outdoor open area test site. The tent is placed at Volvo Cars in Gothenburg, Sweden and has the dimensions $20 \mathrm{~m} \times 12 \mathrm{~m} \times 6 \mathrm{~m}$ $($ length $\times$ width $\times$ height $)$ with a $4 \mathrm{~m}$ diameter turntable inside, see Fig. 3.

The field in front of the reflector was measured by using a planar $3 \mathrm{~m} \times 3 \mathrm{~m}$ metal positioning system with two step motors. The probe antenna used for measuring the field was a Microwave Biconical Antenna (Schwarzbeck SBA 9113, 0.5$3 \mathrm{GHz}$ ), which was mounted in a plastic holder positioned on the positioning grid, see Fig. 3. The positioning grid was centered in front of the reflector antenna. The center position of the grid was placed at distance $d=4 \mathrm{~m}$ from the feed array.

To have an accurate placement of the positioning grid relative to the reflector was a challenge since the floor was not completely level. In addition, there were no accurate structures to center the grid relative to the reflector at the same height and level as the reflector. This was all done by hand with the help of a laser measurer.

The transmission S-parameter, $S_{21}$, between the input port of the measurement antenna and the output port of the probe antenna was measured using a network analyzer (Agilent $8753 \mathrm{ES} 30 \mathrm{kHz}-6 \mathrm{GHz}$ S-parameter Network Analyzer). The network analyzer was placed in a measurement house outside the tent and long radio frequency (RF)-cables were employed to connect it with the measurement system inside the tent. The length of the cable from the network analyzer to the input port of the measurement antenna was $45 \mathrm{~m}$ long (a $35 \mathrm{~m}$ cable plus a $10 \mathrm{~m}$ cable) and the cable from the output port of the probe antenna, back to the network analyzer, was $49 \mathrm{~m}$ long (a $45 \mathrm{~m}$ cable plus a $4 \mathrm{~m}$ cable), with a cable loss of $36.4 \mathrm{~dB} / 100 \mathrm{~m}$. The very long RF-cables make it very hard to accurately calibrate the network analyzer. Instead, the cable losses were measured and accounted for later on in the post-processing. The cable losses were measured for the cable from the network analyzer port to the measurement antenna, as well as the cable from the other network analyzer port to the probe antenna. For higher frequency measurements, one should consider reducing the cable lengths at the measurement site, e.g., by placing the network analyzer closer to the reflector.

The $S_{21}$ was measured over the frequency range from $1 \mathrm{GHz}-3 \mathrm{GHz}$, with 201 samples, corresponding to a frequency resolution of $10 \mathrm{MHz}$. A power amplifier was used in the setup to compensate for the loss of the very long cables.

The field in front of the reflector was probed in a rectangular area positioned at $h=1.4 \mathrm{~m}$, measured from the base of the reflector, corresponding to a height of $1.7 \mathrm{~m}$ from the 


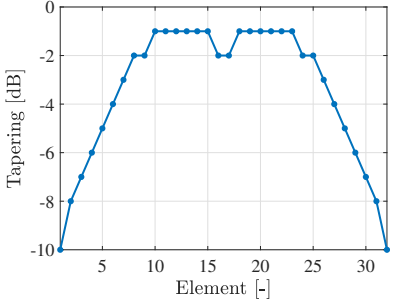

Fig. 4. Amplitude tapering indicated for each element for the tapered feed array.

floor inside the tent. The height $1.7 \mathrm{~m}$ was chosen because it is a typical car roof height, where a typical shark-fin antenna could be placed. The grid area covered a width of $y \in[-1.45 \mathrm{~m}, 1.45 \mathrm{~m}]$ and a depth of $z \in[4 \mathrm{~m}, 7 \mathrm{~m}]$. The grid spacing was $d_{y}=d_{z}=5 \mathrm{~cm}$, corresponding to $\lambda / 2$ at $3 \mathrm{GHz}$. The measurements were done over the grids by sweeping along $y$, at a fixed $z$-position, then moving to the next $z$ position, sweeping along $y$, moving to the next $z$-position etc.

Measurements were also performed for five different heights, $x \in[1.1 \mathrm{~m}, 1.5 \mathrm{~m}]$, with a step of $d_{x}=10 \mathrm{~cm}$. For all the heights, except $x=1.4 \mathrm{~m}$, the measurements were performed along a cross in the middle of the grid area, the same verification procedure as introduced in [27]. In [27] it is shown through simulations that instead of sampling a full grid, one can sample along a cross and get an STD close to the one for the full grid. The cross is located in the center of the grid area, with the first line located at $z=5.5 \mathrm{~m}$ and $y \in[-1.45 \mathrm{~m}, 1.45 \mathrm{~m}]$, and the second line located at $y=0 \mathrm{~m}$ and $z \in[4 \mathrm{~m}, 7 \mathrm{~m}]$. An example of the cross is shown in the test zone circle in Fig. 1. The spacing of the samples in the cross is $d_{y}=d_{z}=5 \mathrm{~cm}$.

As an investigation of improving the field variations, additional measurements were performed using a feed array with amplitude tapered distribution, where the vertical port for each element was connected to an attenuator before being connected to the feeding network. The field variations mainly occur because the reflector and feed array are finite in size. Another source for the variation is the direct radiation from the feed array into the test zone, however, this is reduced by the two added corrugations as described in Section II-C. The amplitude tapering is shown in Fig. 4. The reason that every element was connected to an attenuator is that the uniform phase distribution is desired for the feed array, which we do not get if some elements have attenuators and some not. The phase shift of the different attenuators were measured and for the attenuators ranging from $1 \mathrm{~dB}-10 \mathrm{~dB}$ the phase variation was $\Delta \phi=7^{\circ}-14^{\circ}$ for $1.5 \mathrm{GHz}-3 \mathrm{GHz}$. The amplitude tapered setup was only measured at height $x=1.4 \mathrm{~m}$, with the same grid area as the non-tapered feed array, but with the spacing $d_{y}=5 \mathrm{~cm}$ and $d_{z}=10 \mathrm{~cm}$. A larger grid spacing in the $z$-direction was chosen due to time-constraints.

\section{Field Verification Measurements}

\section{A. Post-processing of Measurements}

In all practical antenna measurement systems there always are unwanted reflections present in the measurement setup.

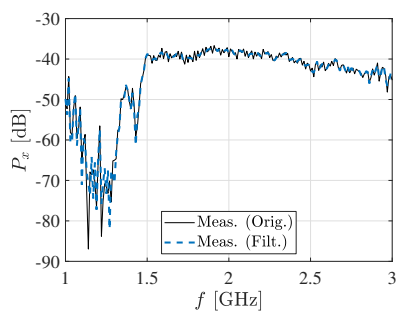

(a)

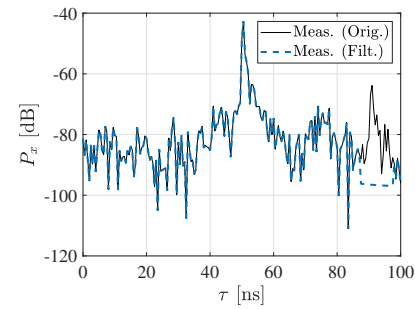

(c) (b)

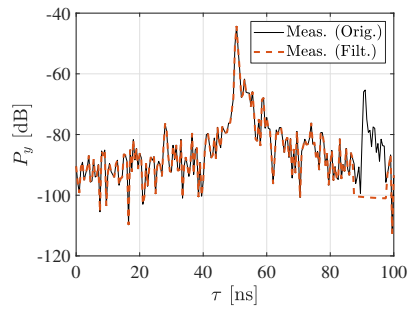

(d)

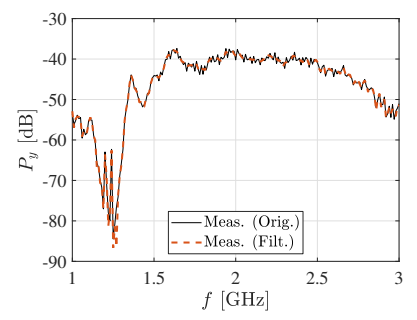

Fig. 5. Frequency and impulse response of the original and filtered measurement data for the vertical $(x)$ and the horizontal $(y)$ polarizations. The results are shown for the grid position with the coordinates $(1.4 \mathrm{~m}, 0 \mathrm{~m}, 5.5 \mathrm{~m})$. (a) Frequency response for the $x$-polarization. (b) Frequnecy response for the $y$ polarization. (c) Impulse response for the $x$-polarization. (d) Impulse response for the $y$-polarization.

These unwanted reflections can be seen in the impulse responses for both polarizations in Fig. 5. The frequency and impulse responses in Fig. 5 are for the non-tapered feed array and the grid position with the coordinates $(1.4 \mathrm{~m}, 0 \mathrm{~m}, 5.5 \mathrm{~m})$. The clear second reflection peak present around $\tau=90 \mathrm{~ns}$ comes from the reflection in the tent with its wooden support structure in front of the reflector.

The reflection coming from the tent was filtered away in the post-processing. The filtering was done in the time-domain (as in time-gating measurements), where the reflection from the tent was removed, and thereafter the processed data was transferred back to the frequency-domain.

The filtering was done for each grid position, by computing a reference impulse response, by taking the inverse Fourier transform of a first order polynomial that was fitted (in a least-square sense) to the absolute value of $S_{21}$. The reference impulse response was then shifted in time, such that the maximum peak was located at the same maximum peak position as for the original impulse response from the $S_{21}$ data. The unwanted tent reflection peak was identified in the impulse response from the $S_{21}$ data for each grid position. The reflection peak could be found through the knowledge of its position relative to the main peak, which varied for the different grid positions. In the filtered impulse response the data around the reflection peak was set to the shifted reference curve values. The difference between the original unfiltered and filtered impulse response is shown in Fig. 5. An interval of $\pm 50 \mathrm{~ns}$ around the peak was set to the reference value. The filtered frequency response was then achieved by taking the filtered impulse response and transferring it back to the frequency domain. 

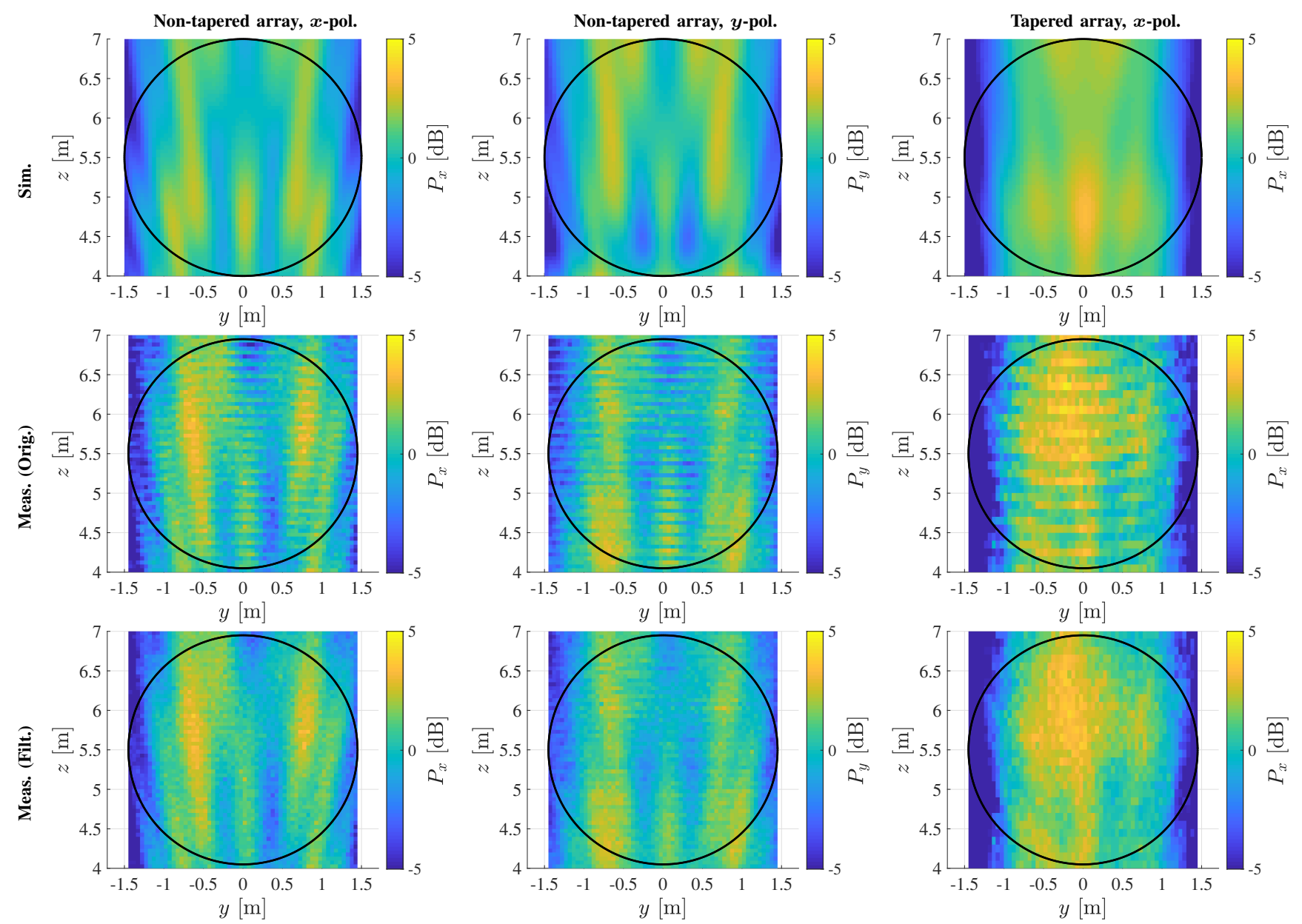

Fig. 6. Normalized power $P$ for the vertical $(x)$ and the horizontal $(y)$ polarizations, in the $y z$-plane at height $h=1.4 \mathrm{~m}$ and $f=2 \mathrm{GHz}$.

\section{B. Test Zone}

The field distribution in front of the reflector was measured as described in Section III-B. The normalized power $P$ variations from the measurements have been plotted together with the corresponding simulated results in Fig. 6. The normalization is done in terms of the average power of the data. The subscripts $x$ and $y$,correspond to the vertical and the horizontal polarizations respectively, see Fig. 1 . The normalized power $P$ is defined as $P_{x}=\left|E_{x}\right|^{2}$ and $P_{y}=\left|E_{y}\right|^{2}$ for the simulated results. Most of the figures in this paper are presented for $2 \mathrm{GHz}$, which was chosen as a representative frequency in the middle of the frequency band. This also complements the simulation results for $2 \mathrm{GHz}$ presented in [22].

Ideally, the generated field should emulate a plane wave, i.e., with constant amplitude and phase on the wavefront. The plane wave propagates with the propagation factor, $e^{-j k z}$, where the wavenumber $k=2 \pi / \lambda$. By removing the theoretical variations of the phase in the propagation direction for both the measured and simulated data it is possible to compare and easier see how the phase, $\phi$, varies in front of the reflector. The phase variations are plotted in Fig. 7.

The normalized power and phase distribution, for the $x$ polarization of the measurement antenna with the tapered feed array, are shown in Fig. 6 and Fig. 7 as well. The measurements for the tapering was only realized for one of the polarizations, the $x$-polarization. Therefore the simulation of the $y$-polarization has been excluded here. However, the simulated results for the tapered feed array in the vertical and the horizontal polarizations are very similar. An analysis of the variations, in terms of STD will be shown in Section IV-C.

In Fig. 6 and Fig. 7, the measured results are presented both as original unfiltered data and filtered data, where the tent reflection was removed. The filtering has been done according to the description in Section IV-A. The figures show the data at the height $h=1.4 \mathrm{~m}$, for the rectangular area $y \in[-1.45 \mathrm{~m}, 1.45 \mathrm{~m}]$ and $z \in[4 \mathrm{~m}, 7 \mathrm{~m}]$.

It can be seen that the measured results are in good agreement with the simulated results, with similar behavior in both Fig. 6 and Fig. 7. In the filtered case we have removed the visible depth variation caused by the reflection from the tent, resulting in a better agreement with the simulated case. It is also visible that we have a smoother field variation for the tapered feed array system, compared to the non-tapered feed array, for both the simulations and the measurements.

In Fig. 8 and Fig. 9, the simulated field variations in terms of power and phase for the $x y$-plane at the distance $d=4 \mathrm{~m}$, are shown respectively. The figures present data for both polarizations and the results are shown for both the nontapered and tapered feed-array. It is clearly visible, especially in Fig. 8 that we have achieved a smoother field variation using 

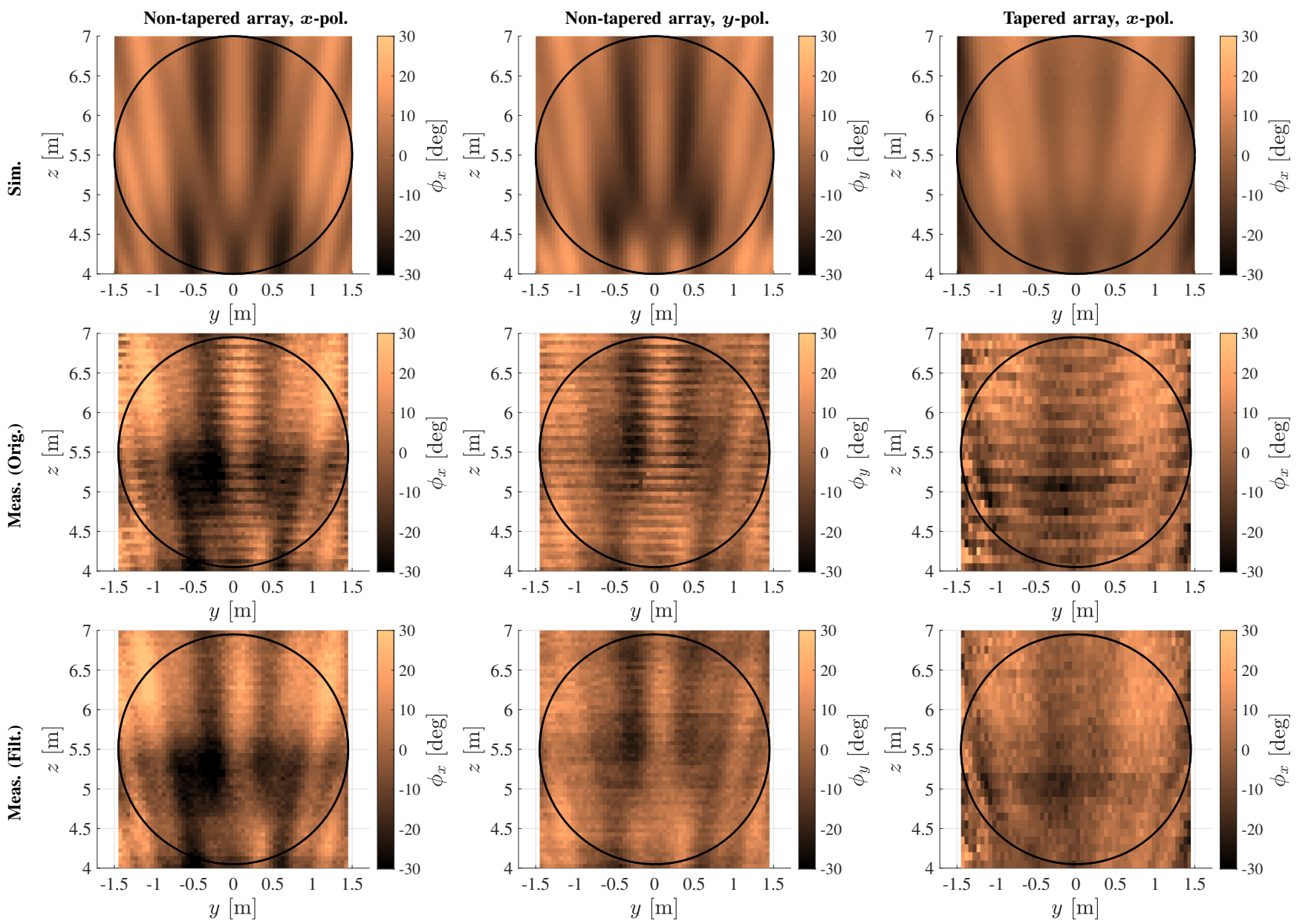

Fig. 7. Normalized phase $\phi$ for the vertical $(x)$ and the horizontal $(y)$ polarizations, in the $y z$-plane at height $h=1.4 \mathrm{~m}$ and $f=2 \mathrm{GHz}$.
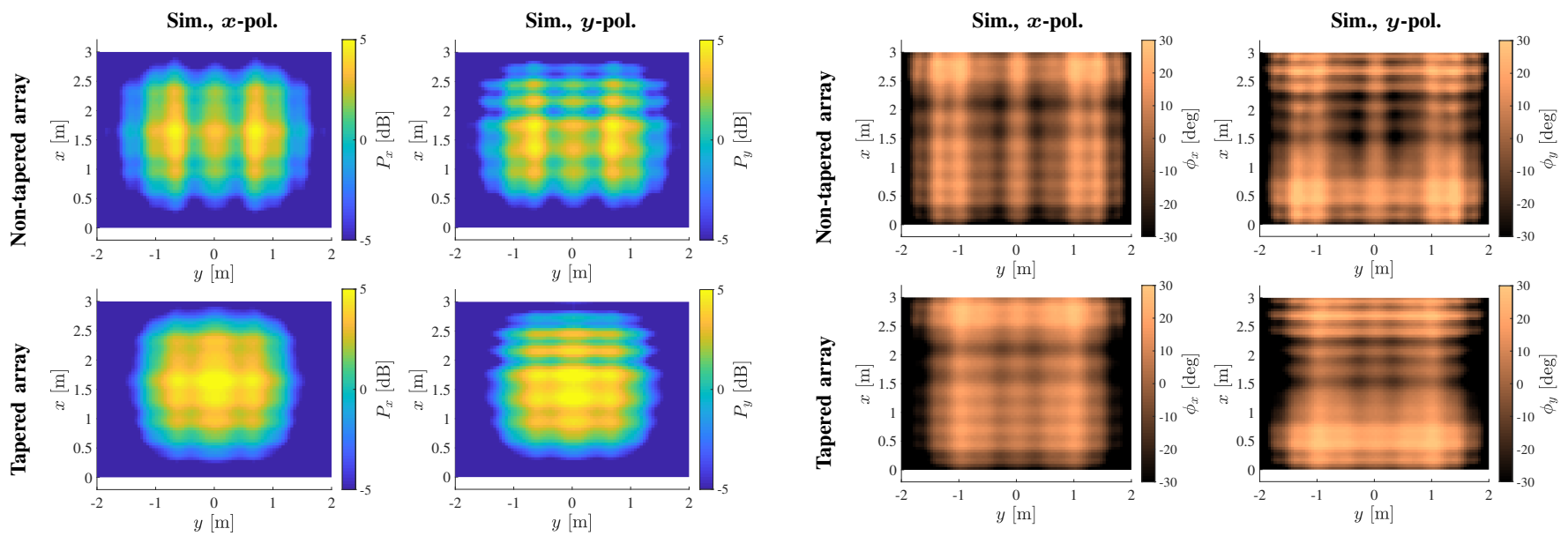

Fig. 8. Simulated normalized power $P$ for the vertical $(x)$ and horizontal $(y)$ polarization, in the $x y$-plane at distance $d=4 \mathrm{~m}$ and $f=2 \mathrm{GHz}$.

the tapered feed array compared to the non-tapered feed array. The ripple, that can be seen for the $y$-polarization in Fig. 8 for larger $x$-values, comes from the back radiation of the feed array.

The field distribution in terms of normalized power $P$ along a line in the $y$-direction at the height $h=1.4 \mathrm{~m}$ and distance $d=4 \mathrm{~m}$ is shown in Fig. 10. The figure shows a comparison
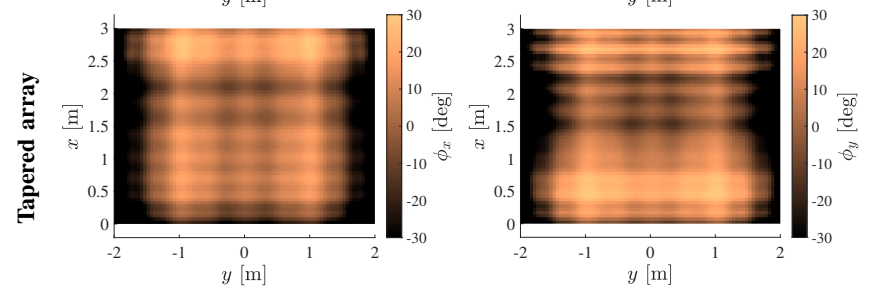

Fig. 9. Simulated normalized phase $\phi$ for the vertical $(x)$ and horizontal $(y)$ polarization, in the $x y$-plane at distance $d=4 \mathrm{~m}$ and $f=2 \mathrm{GHz}$.

between the simulated and the measured data for both the non-tapered feed array and the tapered feed array system. The shape of the simulated and measured curves follow each other well, but with slightly larger variations for the measured data. It can be seen that the simulated peak-to-peak variation in $y \in$ $[-1 \mathrm{~m}, 1 \mathrm{~m}]$ for both the non-tapered and tapered feed array are around $3 \mathrm{~dB}$ with slightly larger variation in the corresponding 

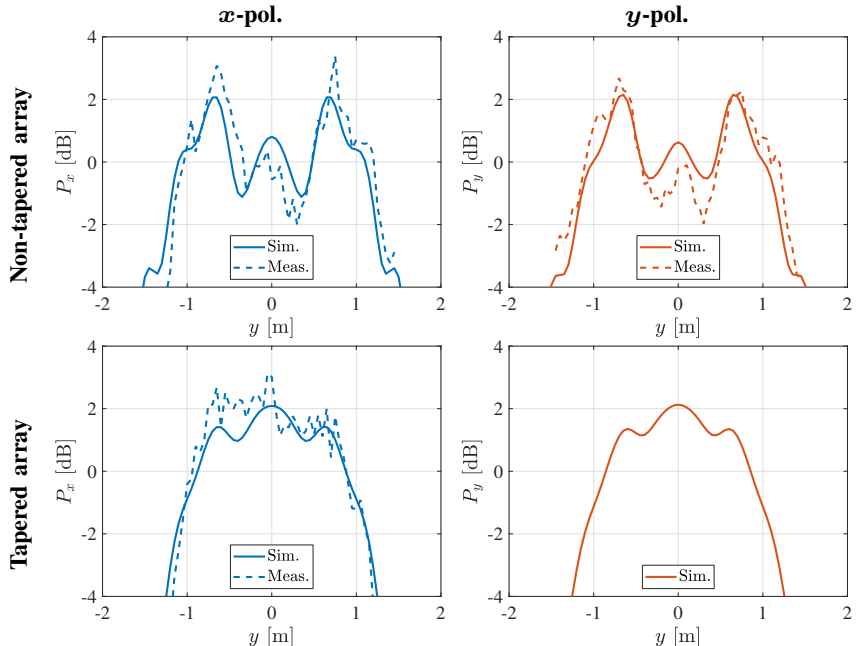

Fig. 10. Simulated and measured normalized power $P$, along a line in the $y$-direction in front of the reflector, at $h=1.4 \mathrm{~m}, d=4 \mathrm{~m}$, for the vertical $(x)$ and horizontal $(y)$ polarization. The results are presented at $2 \mathrm{GHz}$.
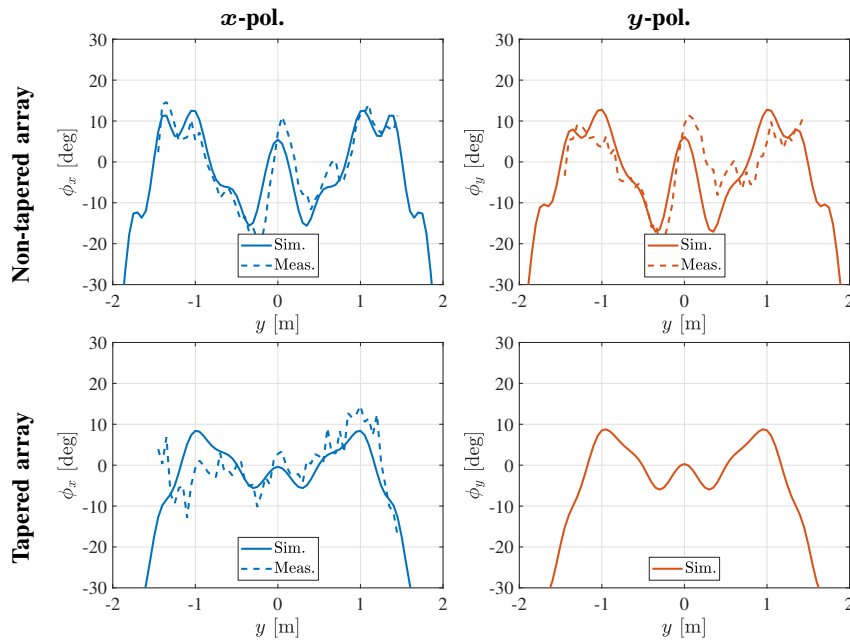

Fig. 11. Simulated and measured field distributions, in terms of normalized phase $\phi$, along a line in the $y$-direction in front of the reflector, at $h=$ $1.4 \mathrm{~m}, d=4 \mathrm{~m}$, for the vertical $(x)$ and horizontal $(y)$ polarization. The results are presented at $2 \mathrm{GHz}$.

measurements. However, if the width is decreased slightly to $y \in[-0.75 \mathrm{~m}, 0.75 \mathrm{~m}]$, then the non-tapered feed array still has a variation of around $3 \mathrm{~dB}$, whereas the system with the tapered feed array performs better and has now a peak-to-peak variation of less than $1.3 \mathrm{~dB}$.

The field distribution in terms of normalized phase $\phi$ along a line in the $y$-direction at the height $h=1.4 \mathrm{~m}$ and distance $d=4 \mathrm{~m}$ is shown in Fig. 11. The figure shows a comparison between the simulated and the measured data for both the non-tapered feed array and the tapered feed array system. The shape of the simulated and measured curves follow each other well. The simulated peak-to-peak variation at $y \in[-1 \mathrm{~m}, 1 \mathrm{~m}]$ is below $30^{\circ}$ for the non-tapered feed array and below $15^{\circ}$ for the tapered feed array. When looking at a smaller width, $y \in[-0.75 \mathrm{~m}, 0.75 \mathrm{~m}]$, the non-tapered feed array has a peakto-peak variation below $25^{\circ}$ and the tapered feed array has a variation of $11^{\circ}$. It is clearly seen that the tapered feed array reduces the peak-to-peak variation.
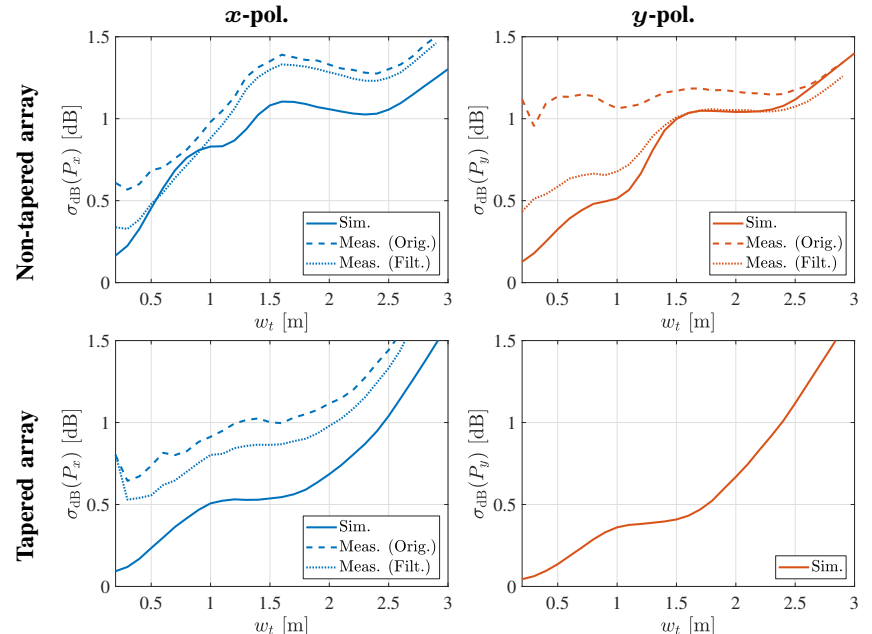

Fig. 12. Simulated and measured STD, $\sigma_{\mathrm{dB}}(P)$, of the normalized power $P$ as a function of the circular test zone diameter $w_{t}$, at $h=1.4 \mathrm{~m}, d=4 \mathrm{~m}$ and $2 \mathrm{GHz}$

\section{Standard Deviation}

To summarize the field variations within the test zone, the STD can be used. The same STD metric was chosen for the random-LOS setup as for the RC setup [28], in order to have a similar figure of merit for both the edge environments stated in the hypothesis in Section I. The STD $\sigma_{\mathrm{dB}}$ of the normalized power $P$ within the test zone has been calculated in $\mathrm{dB}$ according to the formula [28]

$$
\sigma_{\mathrm{dB}}(P)=5 \log \left(\frac{1+\sigma(P)}{1-\sigma(P)}\right)
$$

where $\sigma$ is the STD of the normalized power in linear units. The power is normalized with its mean, to achieve the normalized power used for the STD. The STD of the phase, $\sigma(\phi)$, is calculated for the phase values where the theoretical phase variations in the propagation direction have been removed, as described in Section IV-B.

In Fig. 12 the simulated and measured $\sigma_{\mathrm{dB}}(P)$ are shown as a function of the circular test zone diameter $w_{t}$ at $2 \mathrm{GHz}$. The test zone in this case is defined as a circle in the $y z$-plane, as shown in Fig. 1 and the used data corresponds to the data within the black circles in Fig. 6. A circle is chosen since it corresponds to the area that will be covered using a rotating turntable. The STD is shown for both the vertical and the horizontal polarizations for both the non-tapered and tapered feed array. The corresponding data for the phase, i.e., $\sigma(\phi)$, is shown in Fig. 13.

The measured and the simulated variations shown in Fig. 12 are in good agreement with each other. The curves follow the same trend, however the measurements show a larger fluctuation. For the simulated non-tapered feed array case we can keep an STD of around $1 \mathrm{~dB}$ or less when $w_{t}<2.5 \mathrm{~m}$, the measured data, using vertical polarization results, are $0.3 \mathrm{~dB}$ higher than the simulated data when $w_{t}>1.2 \mathrm{~m}$. However, the filtered data for the horizontal polarization is very close to the simulated one. The simulation data for the tapered feed array gives the same performance in terms of STD as the non-tapered feed array when $w_{t}=2.5 \mathrm{~m}$, but an improved 

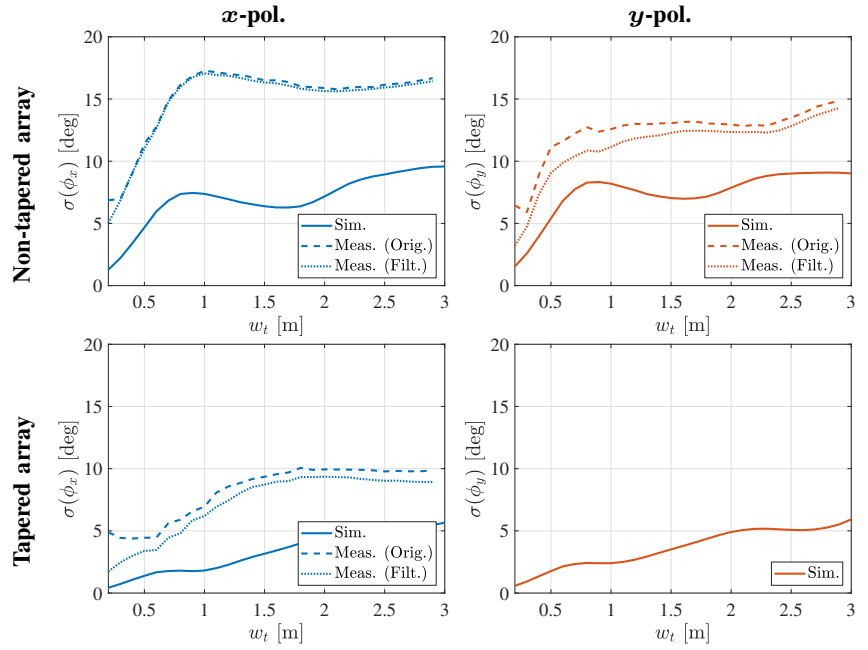

Fig. 13. Simulated and measured STD, $\sigma(\phi)$, of the phase $\phi$ as a function of the circular test zone diameter $w_{t}$, at $h=1.4 \mathrm{~m}, d=4 \mathrm{~m}$ and $2 \mathrm{GHz}$.

performance for widths smaller than that. For $w_{t}=1.5 \mathrm{~m}$ the STD for the tapered feed array is $0.5 \mathrm{~dB}$, while the non-tapered feed array has an STD of $1 \mathrm{~dB}$. For both array types and both polarizations we can see that we get an improvement when filtering the measured data, and thus removing the reflections from the tent. This indicates that a better performance could be expected in an AC.

The phase variations presented in Fig. 13 show a simulated STD of less than $10^{\circ}$ for up to $w_{t}=3 \mathrm{~m}$ in the non-tapered feed array system. For the tapered feed array the STD is reduced to $5^{\circ}$. The measured and simulated data shown in Fig. 11 are also in good agreement with each other. However, the phase variations from the measurements in Fig. 13 are around double that of the simulated data for the vertical polarization. This is probably because the measurements have been performed over several days. During this time the phase drifts and it is hard to accurately measure the phase. This could have been solved by connecting a $10 \mathrm{MHz}$ reference signal to the network analyzer. However, we did not have access to such at the measurement site.

In Fig. 14 and Fig. 15, the simulated and measured $\sigma_{\mathrm{dB}}(P)$ and $\sigma(\phi)$ are shown as a function of frequency, respectively. The results are shown for a fixed test zone diameter of $w_{t}=$ $2 \mathrm{~m}$. The test zone in this case is defined as a circle in the $y z$-plane as shown in Fig. 1. The test zone is located at $h=$ $1.4 \mathrm{~m}$ and $d=4 \mathrm{~m}$. The STD is shown for both the vertical and the horizontal polarizations for both the non-tapered and tapered feed array. The simulated and measured data is based on results for every $100 \mathrm{MHz}$ in the interval $1.5 \mathrm{GHz}-3 \mathrm{GHz}$.

The measured and simulated data presented in Fig. 14 follow each other well, but with a larger variation in the measurement data compared to the simulations. The variation over frequency is quite flat for both the simulated and the measured data, with similar STD. This shows that the same test zone location is suitable for the frequency range from $1.5 \mathrm{GHz}-3 \mathrm{GHz}$. For a different frequency range, employing a different feed array, the suitable test zone location might change; however, this is left for future work. There is a
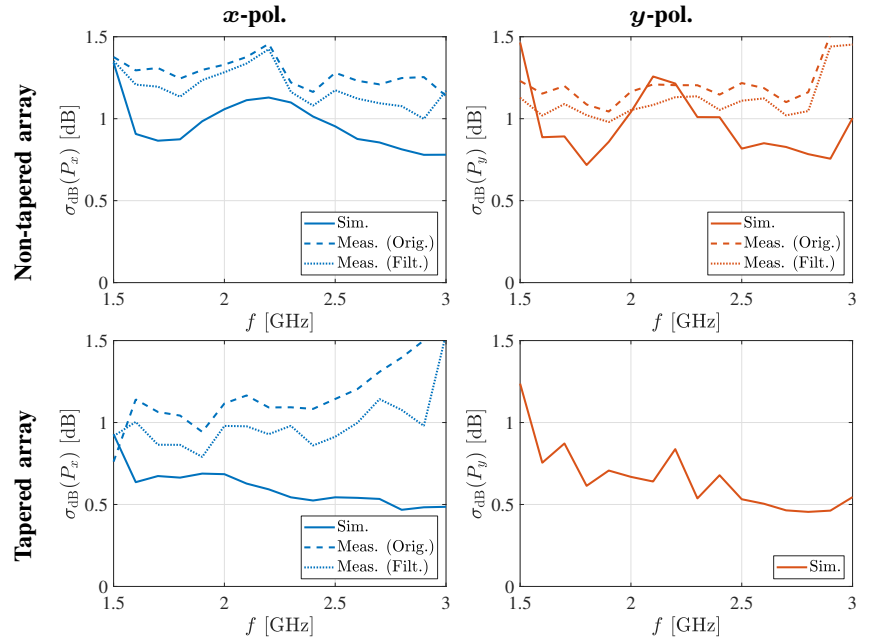

Fig. 14. Simulated and measured STD of the normalized power $P$ as a function of the frequency, for a test zone diameter of $w_{t}=2 \mathrm{~m}$, at $h=1.4 \mathrm{~m}$ and $d=4 \mathrm{~m}$.
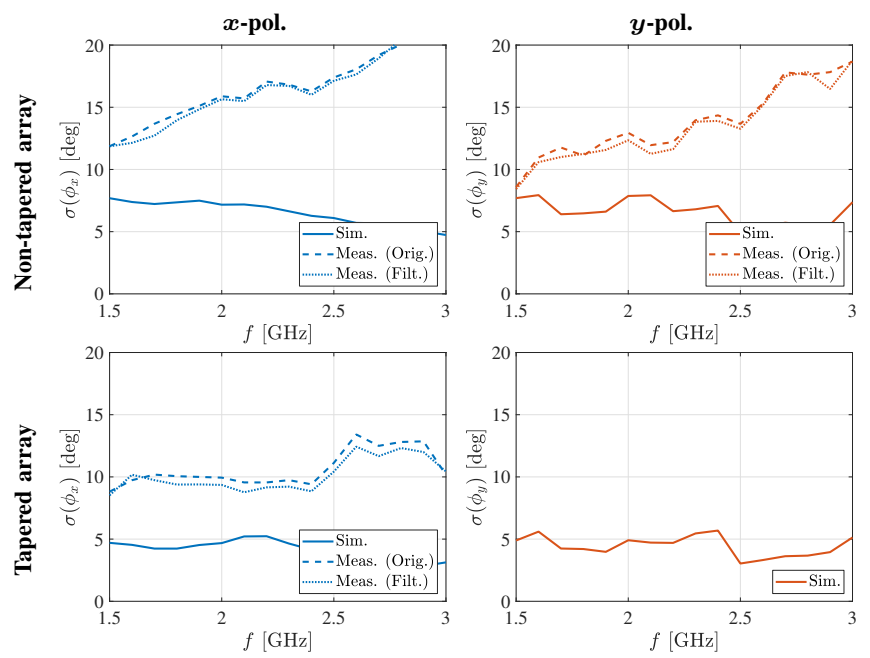

Fig. 15. Simulated and measured STD of the normalized phase $\phi$ as a function of the frequency, for a test zone diameter of $w_{t}=2 \mathrm{~m}$, at $h=1.4 \mathrm{~m}$ and $d=4 \mathrm{~m}$.

trend towards larger STD for the measurements compared to the simulations for the $y$-pol non-tapered feed array and the $x$-pol tapered feed array. It is expected, since the grating lobes that starts to appear close to $3 \mathrm{GHz}$ would affect the measurement more than the simulation results. This is because the simulations are performed in free-space, whereas the measurements are performed in an outdoor tent, where objects, such as the wooden structure of the tent are reflecting the radiation from the grating lobes. How much the grating lobes affect the results depend on the exact placement of the array and the reflector inside the tent. Another explanation for the measured larger STD for the tapered case can be the nonideal attenuators, which have a larger phase variation at the higher frequencies. This introduces a phase difference between the elements and the elements will therefore no longer have uniform phase excitation, which was the assumption in the simulated tapering. This could be solved by using attenuators with less phase variation.

In Fig. 15 the measured results show an increased phase 

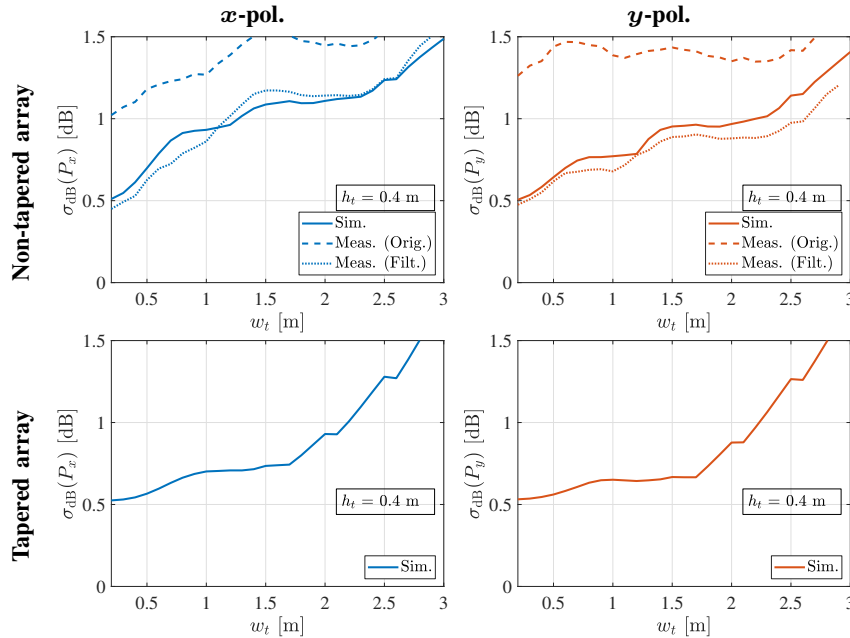

Fig. 16. Measured and simulated STD of the power $P$ within a test zone of height $h_{t}=40 \mathrm{~cm}$, as a function of test zone width at $f=2 \mathrm{GHz}$.

variation for higher frequencies. This is expected, since the measurements were performed over several days, and it becomes harder to accurately measure the phase at higher frequencies. The phase variation of the measured data for the tapered feed array does not increase as much as the for the non-tapered feed array for higher frequencies. This is most likely due to that the tapered feed array measurements were performed in half the time, compared to the non-tapered feed array, because fewer samples were used.

The field variation within a volume is calculated using the measurement data for the five different crosses as described in Section III-B. These variations are summarized in terms of STD as a function of test zone width in Fig. 16. The STD is calculated for a test zone volume of height $h_{t}=0.4 \mathrm{~m}$ at $2 \mathrm{GHz}$. The simulated results are calculated for the same cross grids as the measured data. It can be seen that measured and simulated curves follow each other very well for the measured data where the reflections from the tent have been filtered away. The STD for the volume is similar to the one for the plane, which was presented in Fig. 12. The data is only shown in terms of power and not phase, since the measurements were not done with dense enough spacing to accurately account for the phase variation. However, the simulated $\sigma(\phi)$ for a test volume of height $h_{t}=0.4 \mathrm{~m}$ were $5^{\circ}-10^{\circ}$ for a test zone width of up to $3 \mathrm{~m}$ for both polarizations.

\section{Passive Vehicular Measurements}

The random-LOS measurement system can be used to perform passive radiation pattern measurements of different antennas, among these also car mounted antennas.

\section{A. Measurement Setup}

The passive vehicular measurements presented in this section were performed in the same outdoor open area test site as the field verification measurements presented in Section IV. Due to practical reasons, the measurements were performed using the random-LOS measurement system with the nontapered feed array.
The antenna under test (AUT) was a prototype shark-fin antenna mounted on the roof of a Volvo S90 at the intended operation position of the antenna. The antenna is a part of the car body and therefore they shall not be separated in the radiation pattern measurements or any other OTA measurement. However, they shall be removed and replaced by an antenna with known gain to perform reference measurements in the test zone.

The transmission, $S_{21}$, measurements were performed by using the same network analyzer as in the field characterization measurements. The measurements were performed over the frequency range $1 \mathrm{GHz}-3 \mathrm{GHz}$ using 201 samples, corresponding to a $10 \mathrm{MHz}$ frequency step, using the vertical polarization. The turntable was rotated continuously, and the speed was adjusted in order to get at least 360 samples in a whole turn. The measurements were filtered in the same way as described in Section IV-A, where the reflection in the tent with its supporting wooden structure was removed from the measurement data.

\section{B. Reference Measurement}

A reference measurement was performed in order to get absolute gain values for the radiation pattern measurement. Therefore, an antenna with known gain was used. For this purpose the same biconical antenna (Schwarzbeck SBA 9113, $0.5-3 \mathrm{GHz}$ ) used for the field verification measurements was employed. The antenna was placed in the center of the turntable, directed towards the reflector, at a distance of $5.5 \mathrm{~m}$.

\section{Radiation Pattern Measurements of Shark-fin Antenna}

The measured radiation pattern of the AUT at $2 \mathrm{GHz}$ using the random-LOS measurement setup can be seen in Fig. 17. Zero degrees corresponds to the back of the car pointing towards the random-LOS reflector. The measured pattern is compared to reference data from measurements performed at a near-field to far-field (NF/FF) antenna measurement range. The NF/FF measurements were performed a year before and the details regarding the measurements are not available at this time.

The measured radiation pattern fits well to the reference for the angles corresponding to the front and back of the car, however to the side, $+90^{\circ}$, it differs more. This is because the AUT on the car was offset with a radius of $1.5 \mathrm{~m}$ to the center of the turntable. The reflector was also not perfectly centered relative to the center of the turntable, which caused the AUT to move outside of the test zone on one of the sides.

The mean squared error (MSE) for the radiation pattern measured with the NF/FF and random-LOS setup was calculated according to

$$
\mathrm{MSE}=\frac{1}{n} \sum_{i=1}^{n}\left(X_{i}-Y_{i}\right)^{2},
$$

where $X_{i}$ and $Y_{i}$ are the NF/FF and random-LOS absolute linear values of the radiation pattern, respectively. The sum is taken over all the $n$ angles for the radiation pattern. When including all the measured angles, $0^{\circ}-360^{\circ}$, the MSE 


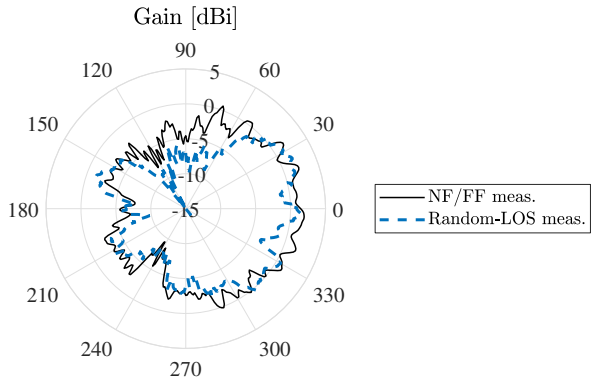

Fig. 17. Measured radiation pattern using the random-LOS system for a roof-mounted prototype shark-fin antenna on a Volvo $\mathrm{S} 90$ at $2 \mathrm{GHz}$.

becomes $3.7 \%$. When excluding the angles in the interval $+45^{\circ}$ to $135^{\circ}$, a MSE value of $1.7 \%$ is achieved.

\section{Active Vehicular Measurements}

The random-LOS measurement system can be used to perform active vehicular OTA measurements. The active vehicular measurements presented in this section are done in the same outdoor tent as the field verification measurements presented in Section IV and the passive vehicular measurements in Section V. For practical reasons the non-tapered feed array, with equal amplitude for all elements, was used for the active measurements.

\section{A. Measurement Setup}

The schematic view of a general active measurement can be seen in Fig. 18, as well as a picture of the actual measurement setup in Fig. 19.

The active measurements require a control computer, from where the communication tester and the turntable are controlled, and where the measurement data is collected, see Fig. 18. However, in the active measurements performed here the turntable was controlled manually. The communication tester (R\&S CMW 500 Wideband Radio Communication Tester, 1201.0002K50) is connected to the two ports on the measurement antenna. In order to get high enough power to run a 64-quadrature amplitude modulation (QAM) modulation scheme, two power amplifier (PA) (Mini-Circuits Amplifier, ZVE-8G and ZVE-8G+, $2000-8000 \mathrm{MHz}$ ), one for each downlink were used.

The DUT for the measurements was a vehicle (Volvo V60), tested with four different roof-mounted antennas. The antennas, mounted one at the time in the position at the back of the roof, where normally a shark-fin antenna is located, was connected to an LTE modem. The four different roof-mounted antennas were four of the two-port MIMO reference antennas defined in [29]. The antennas Good and Nominal for LTE band 2 (downlink (DL) $f=1930-1990 \mathrm{MHz}$ ) and band 7 (DL $f=2620-2690 \mathrm{MHz}$ ) were used. However, the car modem supported the European LTE bands, and instead measurements were performed using LTE band 3 (DL $f=1805-1880 \mathrm{MHz}$ ) and 7. The difference in performance between LTE band 2 and 3 for the antennas were assumed to be negligible, due to the closeness in frequency. The vehicle including the roofmounted antennas are hereafter called DUT A (LTE Band 2
Nominal), DUT B (LTE Band 2 Good), DUT C (LTE Band 7 Nominal) and DUT D (LTE Band 7 Good).

The location of the MIMO reference antenna on the car and the car placement on the turntable meant that the MIMO reference antenna was moving with a radius of $1.65 \mathrm{~m}$ relative to the center of the turntable. This corresponds to a simulated STD within the test zone of $1.5 \mathrm{~dB}$ and $1.6 \mathrm{~dB}$ for the vertical and the horizontal polarization, respectively. However, the STD of the repeatability for the active measurement is left for future work.

The $2 \times 2$ MIMO downlink data throughput (TPUT) measurements using transmission mode 3 , were performed on LTE bands 3 and 7, on the channels 1575 (downlink $f=1842.5 \mathrm{MHz}$ ) and 3100 (downlink $f=2655 \mathrm{MHz}$ ), respectively.

A separate uplink is often required in active OTA measurements, such that the communication tester can manage to detect the received signal. In this paper, the separate uplink made use of a dual-polarized bowtie antenna element [26], [30], where only the vertically polarized port was in use. The uplink also required a low noise amplifier (LNA) (MITEQ, AMF-2D-02000600-23-13P, $2000-6000 \mathrm{MHz}$ ) in order for the communication tester to detect the signal.

For all the measurements the reference measurement channels (RMC) were used. The downlink measurement settings were as follow, modulation 64-QAM, $10 \mathrm{MHz}$ cell bandwidth, 50 resource blocks, transport block size (TBS) index 18 and 2000 subframes. These settings give a maximum TPUT in the downlink of $35.424 \mathrm{Mbit} / \mathrm{s}$. The uplink settings were the following, quadrature phase shift keying (QPSK) modulation, $10 \mathrm{MHz}$ cell bandwidth, 50 resource blocks and TBS index 6 .

The TPUT measurements were performed by rotating the vehicle on the turntable, and for every $5^{\circ}$ on the turntable we make a stop and sweep the power (from high to low). For every power level we measure the TPUT level, which means that we will get a TPUT curve going from full TPUT to zero TPUT for every rotation angle of the turntable. By taking the average of all these TPUT curves the overall device performance can be established.

\section{B. Reference Measurements}

Reference measurements were performed to determine the external losses in the setup, in order to get correct TPUT power levels for the receiver. The reference measurements were done with an antenna with known gain. The same biconical antenna (Schwarzbeck SBA 9113, $0.5-3 \mathrm{GHz}$ ) used as a probe in the field characterization was used as the reference antenna. The setup for the reference measurement was similar to the downlink setup in Fig. 18, but instead of a communication tester a network analyzer was used (the same as in the field verification measurements) to measure the transmission coefficient. The biconical reference antenna was centered on the turntable and placed at the $1.5 \mathrm{~m}$ height from the floor, without the car. Two reference measurements were done, one for vertical and one for horizontal polarization.

The reference measurements were used in order to get the external attenuation values that were loaded into the 


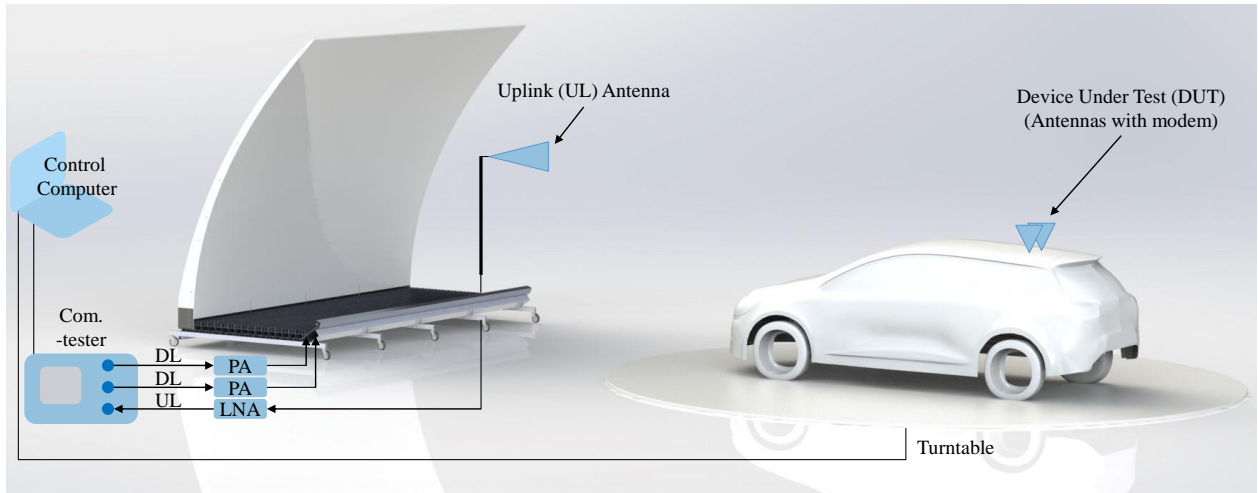

Fig. 18. Schematic view of a general $2 \times 2$ MIMO measurement setup for vehicles.

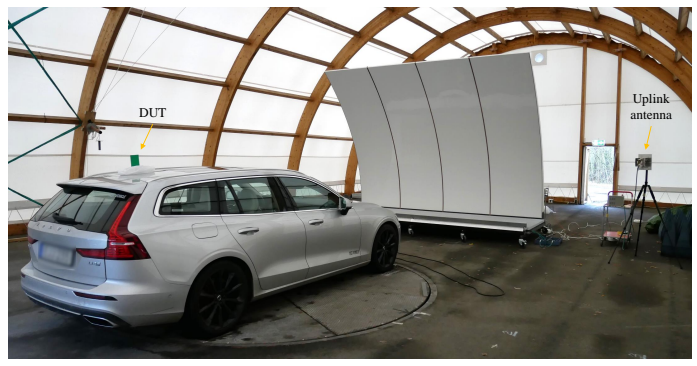

Fig. 19. Picture of the $2 \times 2$ MIMO measurement setup for the Volvo V60. A MIMO reference antenna is mounted in the position where a shark-fin antenna normally is located.

communication tester. The external attenuation values in the downlink include all the attenuation from the output port of the communication tester to the AUT on the vehicle. This means that it includes the cables, the PAs, the measurement antenna and the free-space attenuation to the AUT.

\section{Theoretical Comparison}

A theoretical comparison was made by measuring the radiation patterns of both ports of the roof-mounted MIMO reference antennas. The pattern was measured for all the combination of the two ports of the MIMO reference antenna and the two ports of the measurement antenna, in total giving four patterns for each MIMO reference antenna. The radiation patterns were measured by using the network analyzer connected to the AUT on the vehicle in a passive measurement setup. The passive measurement setup included an LNA (MiniCircuits Low Noise Amplifier, ZX60-83LN, 500-8000 MHz) to improve the signal to noise ratio (SNR). This will in principle give the channel matrix $\mathbf{H}$. In a frequency flat MIMO channel, the signal model is given by $\mathbf{y}=\mathbf{H x}+\mathbf{n}$, where $\mathbf{y}$ is the received signal vector, $\mathbf{H}$ is the channel matrix, $\mathbf{x}$ is the transmit signal and $\mathbf{n}$ is the noise vector [31]. Since the spatial multiplexing scheme used in the receiver mode of the modem was unknown, we have used the zero forcing (ZF) receiver in our theoretical investigation as a typical multiplexing scheme to achieve a theoretical approximation of the active measurements.

The ZF receiver decouples the matrix channel into $N_{t}$ (number of transmit antennas) parallel scalar channels with additive noise. The SNR of the $i$-th data stream using ZF is given by

$$
\gamma_{i}^{\mathrm{ZF}}=\frac{\gamma_{t}}{N_{t}\left[\left(\mathbf{H}^{H} \mathbf{H}\right)^{-1}\right]_{i, i}}
$$

where $\gamma_{t}$ is the transmit SNR, the subscript ${ }^{H}$ stands for the conjugate transpose and $[\mathbf{X}]_{i, i}$ denotes the $i$-th diagonal entry of the matrix $\mathbf{X}$ [31]. Assuming unit variance noise, we can exchange the SNR in Eq. (3) to the power.

The radiation pattern measurements were used to get the $\mathbf{H}$ matrix for every rotation angle on the turntable. Then, the $\gamma_{i}^{\mathrm{ZF}}$ was calculated for every H-matrix. Since $2 \times 2$ MIMO was wanted, the worst of the two diagonal components was chosen as corresponding to the ZF performance. The ZF TPUT was computed using the ideal digital threshold receiver model [32], where the receiver goes from zero TPUT to maximum TPUT at what is described as the threshold level. As the channel changes due to the rotation of the vehicle, the threshold changes, at which the TPUT jumps. Since we assume the ZFreceiver, (3) is used to compute the threshold level for each separate data bitstream. The method presented in [33] is then used to obtain the MIMO TPUT. All the computed threshold levels, i.e., one for each angle, has been combined by taking the cumulative distribution function $(\mathrm{CDF})$ to get the relative TPUT curve for the ZF data.

\section{Active $2 \times 2$ MIMO Measurements on Car}

The active $2 \times 2$ MIMO measurements on the V60 comprised four different antennas mounted on the car. The relative TPUT results for LTE band 3 and 7 are shown in Fig. 20. The solid lines in the figure correspond to the measured TPUT with the DUTs including the modem, whereas the dotted lines correspond to the TPUT calculated using ZF receiver, applied to the measured radiation pattern data. All the ZF curves have been normalized with the same value. The normalization value is calculated from the difference between the power level of the median value for the ZF curve for AUT $A$ and the powerlevel for the $50 \%$ relative TPUT level for the same DUT. The same normalization value is used for all antennas, however, for the band 7 DUTs the power level has been compensated for the gain difference of the reflector and LNA at the different frequencies. 


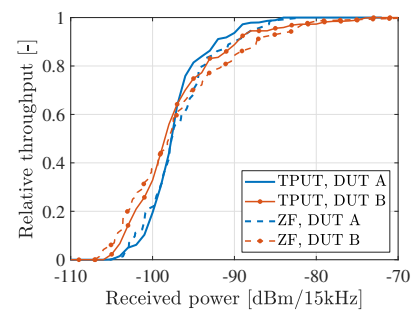

(a)

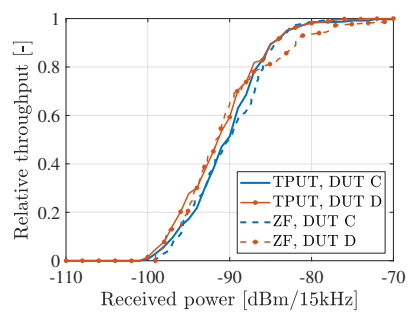

(b)
Fig. 20. Normalized measured MIMO TPUT performance at LTE band 3, (a), and LTE band 7, (b). The solid lines are the measured average TPUT performance, whereas the dotted lines are the normalized computes TPUT curves after applying $\mathrm{ZF}$ to the corresponding measured radiation patterns.

The ZF curves and the measured TPUT curves show similar shape and behavior. The slight difference could be due to that the receiver in the modem uses a different type of spatial multiplexing scheme than what we have assumed. It is not possible to distinguish between the MIMO reference antennas Nominal and Good, as one would expect [29]. This is due to that the performance only in the horizontal plane is measured, where there is not a big radiation performance difference between the compared antennas. The downlink in the system goes on the vertically and horizontally polarized ports, while the two receiving antennas are vertically polarized, this means that the performance is related to the cross polarization level of the receive antennas.

\section{CONCLUSIONS}

The first random-LOS measurement setup for $1.5 \mathrm{GHz}-$ $3 \mathrm{GHz}$ frequencies has been manufactured and characterized. The simulated field variations for the setup compare very well to the measured field variations and a measured STD of $1.3 \mathrm{~dB}$ can be achieved within a circular test zone of $2 \mathrm{~m}$ diameter. The improvement obtained with the feed array with amplitude tapering has also been verified. Indeed, the measured STD of the power variations was reduced to $1 \mathrm{~dB}$ for the $2 \mathrm{~m}$ test zone width. However, it is worthwhile to note that the conditions at the outdoor range measurement facility were not optimal. Hence, a better accuracy of the performance of the system, i.e., a performance closer to the simulated ones, could be expected in a more controlled measurement environment.

The simulated and the measured power variations are in good agreement with each other. However, the measured phase variations are much larger than the simulated ones. This could be explained by the difficulty in measuring the phase accurately, especially in an outdoor environment with very long cables and measurements being performed over several days. An alternative, to achieve more accurate results, would be to perform the measurements in a more controlled environment, such as a semi-AC. However, this would increase the cost significantly, since a large chamber would be needed, whereas an outdoor facility is more accessible and practical in many cases. The system can be used in already existing electromagnetic compatibility (EMC) chambers; however, this would also increase the cost, since the availability might be limited. The requirements on the accuracy of the measurements, versus the cost should be considered in every user case.

A description of how active OTA measurements can be performed using the random-LOS measurement setup has been presented as well as results showing the first $2 \times 2 \mathrm{MIMO}$ TPUT measurements on a vehicle. The tested antennas were four different dual-element roof-mounted MIMO reference antennas, mounted in the place where usually a shark-fin antenna is located. The MIMO reference antennas were mounted on a Volvo V60. The measured TPUT data show good agreement to the calculated TPUT using ZF receiver on the measured radiation pattern.

\section{ACKNOWLEDGMENTS}

This project is partly supported by a grant from the Swedish Research Council VR, through an industrial PhD project, project "Testmiljö för framtidens kommunikationssystem" (D0571801). The work is partly carried out within SIVERT and supported by Fordonsstrategisk forskning och innovation, FFI, and Sweden's Innovation Agency. The authors would like to thank Björn Bergqvist for all his help with the practical work related to the measurements.

\section{REFERENCES}

[1] W. Fan, F. Sun, J. Ø. Nielsen, X. Carreño, J. S. Ashta, M. B. Knudsen, and G. F. Pedersen, "Probe selection in multiprobe OTA setups," IEEE Transactions on Antennas and Propagation, vol. 62, no. 4, pp. 21092120, Apr. 2014.

[2] W. Fan, P. Kyösti, J. O. Nielsen, and G. F. Pedersen, "Wideband MIMO channel capacity analysis in multiprobe anechoic chamber setups," IEEE Transactions on Vehicular Technology, vol. 65, no. 5, pp. 2861-2871, May 2016.

[3] P.-S. Kildal, C. Orlenius, and J. Carlsson, "OTA testing in multipath of antennas and wireless devices with MIMO and OFDM," Proceedings of the IEEE, vol. 100, no. 7, pp. 2145-2157, Jul. 2012.

[4] A. Hussain, P.-S. Kildal, and A. A. Glazunov, "Interpreting the total isotropic sensitivity and diversity gain of LTE-enabled wireless devices from over-the-air throughput measurements in reverberation chambers," IEEE Access, vol. 3, pp. 131-145, 2015.

[5] W. Yu, Y. Qi, K. Liu, Y. Xu, and J. Fan, "Radiated two-stage method for LTE MIMO user equipment performance evaluation," IEEE Transactions on Electromagnetic Compatibility, vol. 56, no. 6, pp. 1691-1696, Dec. 2014.

[6] M. G. Nilsson, P. Hallbjörner, N. Arabäck, B. Bergqvist, T. Abbas, and F. Tufvesson, "Measurement uncertainty, channel simulation, and disturbance characterization of an over-the-air multiprobe setup for cars at $5.9 \mathrm{GHz}$," IEEE Transactions on Industrial Electronics, vol. 62 , no. 12, pp. 7859-7869, Dec. 2015.

[7] CTIA Certification Program, "Test plan for wireless large-form-factor device over-the-air performance," Certification Program Test Plan 1.2.1, Feb. 2019

[8] W. Fan, P. Kyösti, L. Hentilä, and G. F. Pedersen, "MIMO terminal performance evaluation with a novel wireless cable method," IEEE Transactions on Antennas and Propagation, vol. 65, no. 9, pp. 48034814, Sep. 2017.

[9] P.-S. Kildal and J. Carlsson, "New approach to OTA testing: RIMP and pure-LOS reference environments and a hypothesis," in 2013 7th European Conference on Antennas and Propagation (EuCAP), Apr. 2013, pp. 315-318.

[10] P.-S. Kildal, Foundations of Antenna Engineering - A Unified Approach for Line-of-Sight and Multipath, 2015th ed. Gothenburg, Sweden: Kildal Antenn AB, Mar. 2017, available at www.kildal.se.

[11] C. S. P. Lötbäck, A. Skårbratt, and K. Arvidsson, "Over-the-air testing of LTE-advanced features using reverberation chamber," in 2017 11th European Conference on Antennas and Propagation (EUCAP), Mar. 2017, pp. 623-627. 
[12] C. S. P. Lötbäck, A. Skårbratt, and C. Orlenius, "Extending the reverberation chamber using a channel emulator for characterisation of overthe-air performance of multiple-input-multiple-output wireless devices," IET Science, Measurement Technology, vol. 9, no. 5, pp. 555-562, 2015.

[13] M. Barazzetta, D. Micheli, L. Bastianelli, R. Diamanti, M. Totta, P. Obino, R. Lattanzi, F. Moglie, and V. M. Primiani, "A comparison between different reception diversity schemes of a 4G-LTE base station in reverberation chamber: A deployment in a live cellular network," IEEE Transactions on Electromagnetic Compatibility, vol. 59, no. 6 , pp. 2029-2037, Dec. 2017.

[14] D. A. Hill, "A numerical method for near-field array synthesis," IEEE Transactions on Electromagnetic Compatibility, vol. EMC-27, no. 4, pp. 201-211, Nov. 1985.

[15] J. Hansen, Spherical Near-field Antenna Measurements, ser. Electromagnetic Waves Series. London, U.K.: Peter Peregrinus Ltd, 1988, no. 26.

[16] R. Haupt, "Generating a plane wave in the near field with a planar array antenna," Microwave Journal, International ed.; Dedham, vol. 46, no. 9 , pp. 152-166, Sep. 2003.

[17] P.-S. Kildal, "Methods and apparatuses for testing wireless communications to vehicles," Jan. 2014, patent application number PCT/EP2014/054620. Applicant is Kildal Antenn AB.

[18] M. S. Kildal, J. Kvarnstrand, J. Carlsson, A. A. Glazunov, A. Majidzadeh, and P.-S. Kildal, "Initial measured OTA throughput of 4G LTE communication to cars with roof-mounted antennas in 2D random-LOS," in 2015 International Symposium on Antennas and Propagation (ISAP), Nov. 2015, pp. 1-4.

[19] A. A. Glazunov, A. Razavi, and P.-S. Kildal, "Simulations of a planar array arrangement for automotive random-LOS OTA testing," in 2016 10th European Conference on Antennas and Propagation (EuCAP), Apr. 2016, pp. $1-5$.

[20] A. Razavi, A. A. Glazunov, P.-S. Kildal, and R. Maaskant, "Array-fed cylindrical reflector antenna for automotive OTA tests in random line-ofsight," in 2016 10th European Conference on Antennas and Propagation (EuCAP), Apr. 2016, pp. 1-4.

[21] M. S. Kildal, A. A. Glazunov, J. Carlsson, and A. Majidzadeh, "Evaluation of a simplified random-LOS measurement setup for characterizing antennas on cars," in 2017 11th European Conference on Antennas and Propagation (EUCAP), Mar. 2017, pp. 3007-3011.

[22] A. Razavi, A. A. Glazunov, S. M. Moghaddam, R. Maaskant, and J. Yang, "Characterization method of an automotive random-LOS OTA measurement setup," Progress In Electromagnetics Research C, vol. 2018, no. 84, pp. 47-60, 2018.

[23] M. S. Kildal, J. Carlsson, and A. A. Glazunov, "Measurements and simulations for validation of the random-LOS measurement accuracy for vehicular OTA applications," IEEE Transactions on Antennas and Propagation, vol. 66, no. 11, pp. 6291-6299, Nov. 2018.

[24] R. Johnson, H. Ecker, and R. Moore, "Compact range techniques and measurements," IEEE Transactions on Antennas and Propagation, vol. 17, no. 5, pp. 568-576, Sep. 1969.

[25] C. Parini, Theory and Practice of Modern Antenna Range Measurements, ser. IET Electromagnetic Waves Series. London, U.K.: The Institution of Engineering and Technology, Dec. 2014.

[26] S. M. Moghaddam, A. A. Glazunov, and J. Yang, "Wideband dualpolarized linear array antenna for random-LOS OTA measurement," IEEE Transactions on Antennas and Propagation, vol. 66, no. 5, pp. 2365-2373, May 2018.

[27] M. S. Kildal, A. Razavi, J. Carlsson, and A. A. Glazunov, "Test zone verification procedures in a random-LOS measurement setup," in 2019 13th European Conference on Antennas and Propagation (EuCAP), Mar. 2019, pp. $1-5$.

[28] P.-S. Kildal, X. Chen, C. Orlenius, M. Franzén, and C. Patané, "Characterization of reverberation chambers for OTA measurements of wireless devices: Physical formulations of channel matrix and new uncertainty formula," IEEE Transactions on Antennas and Propagation, vol. 60, no. 8, pp. 3875-3891, Aug. 2012.

[29] I. Szini, B. Yanakiev, and G. F. Pedersen, "MIMO reference antennas performance in anisotropic channel environments," IEEE Transactions on Antennas and Propagation, vol. 62, no. 6, pp. 3270-3280, Jun. 2014, conference Name: IEEE Transactions on Antennas and Propagation.

[30] "Bowtie antenna data sheet," Gapwaves AB, Gothenburg, Sweden, Data Sheet, 2017.

[31] A. Paulraj, R. Nabar, and D. Gore, Introduction to Space-Time Wireless Communications. Cambridge University Press, May 2003.

[32] P.-S. Kildal, A. Hussain, X. Chen, C. Orlenius, A. Skårbratt, J. Åsberg, T. Svensson, and T. Eriksson, "Threshold receiver model for throughput of wireless devices with MIMO and frequency diversity measured in reverberation chamber," IEEE Antennas and Wireless Propagation Letters, vol. 10, pp. 1201-1204, 2011.

[33] A. A. Glazunov, S. M. Moghaddam, and J. Yang, "Simulating the MIMO efficiency of antennas," in 2017 International Symposium on Antennas and Propagation (ISAP), Oct. 2017, pp. 1-2.

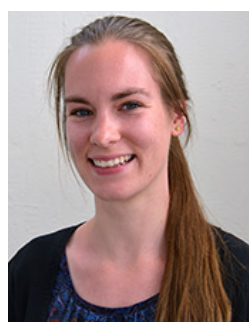

Madeleine Schilliger Kildal was born in Trondheim, Norway, in 1987. She received a Master of Science in Engineering Physics from the Faculty of Engineering at Lund University, Lund, Sweden, in 2013. In 2020 she received a Ph.D. degree from Chalmers University of Technology, Gothenburg, Sweden. Her Ph.D. research covers over-the-air characterization of antenna systems and wireless devices.

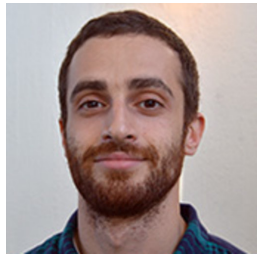

Sadegh Mansouri Moghaddam received the B.Sc. degree in electrical engineering from the University of Guilan, Rasht, Iran, in 2008, and the M.Sc. degree in communication from the $\mathrm{K}$. N. Toosi University of Technology, Tehran, Iran, in 2011. In 2019 he received a Ph.D. degree from Chalmers University of Technology, Gothenburg, Sweden. His Ph.D. research include ultrawideband antennas, phased array antennas, millimeter-wave planar array antennas, multiple-input multiple-output antennas, and overthe-air characterization of antenna systems and wire-

less devices.

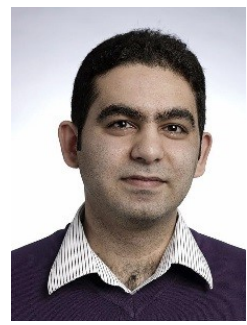

Aidin Razavi was born in 1982. He received the M.Sc. degree in microwave engineering from Tarbiat Modares University, Tehran, Iran, in 2007 and the $\mathrm{Ph} . \mathrm{D}$. degree from Chalmers University of Technology, Gothenburg, Sweden, in 2016. Between 2007 and 2009, he was with HT Telecom Co., Iran as senior engineer, and from 2009 to 2011 with Huawei Technologies Co., as radio network planning and optimization engineer. Since 2017, he has been with Ericsson Research, Ericsson AB, Gothenburg, Sweden. His major areas of research include antenna theory, OTA measurements, MIMO antenna system performance, and optimal antenna apertures. 


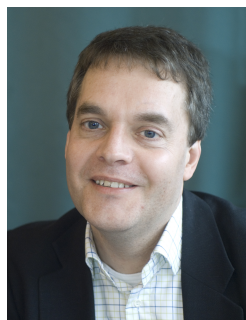

Jan Carlsson (M'98, SM'17) was born in Gothenburg, Sweden, in 1962. He received the M.S.E.E. and Ph.D. degrees from Chalmers University of Technology, Gothenburg, Sweden, in 1986 and 1998, respectively.

From 1986 to 1990, he was an Electromagnetic Compatibility (EMC) Engineer at Ericsson Radar Electronics AB, Mölndal, Sweden. From 1990 to 2016 he was the Head of Research with the EMC Department, SP Technical Research Institute of Sweden, Borås, Sweden. Since 2003, he has been an Adjunct Professor at the Department of Electrical Engineering, Division of Communications, Antennas, and Optical Networks, Chalmers University of Technology. From 2009 to 2016 he was an Assistant Centre Manager with Chase, Chalmers Antenna Systems VINN Excellence Centre. He has over 25 years of research experience covering EMC, computational electromagnetics, antennas, wireless communications, and microwave measurements. He has an extensive experience as Project Leader of national and international research and collaboration projects. He is currently a Senior Researcher with Provinn $\mathrm{AB}$, Gothenburg, where he is responsible for the research activities. He has authored or co-authored more than 170 peer-reviewed papers and contributed to several book chapters.

Dr. Carlsson was the Chairman of the Swedish IEEE EMC Chapter from 2002 to 2004. He is currently the Chairman of Section A (Electromagnetic Metrology) of the Swedish National Committee for Radio Science, and a member of Section E (Electromagnetic Environment and Interference). He is also Chairman of the International Steering Committee of the conference series EMC Europe.

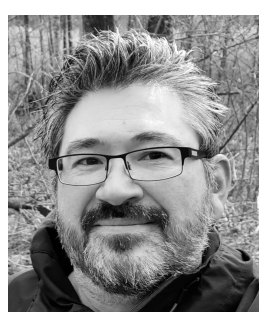

Andrés Alayón Glazunov (SM'11) was born in Havana, Cuba. He received the M.Sc. (EngineerResearcher) degree in physical engineering from Peter the Great St. Petersburg Polytechnic University (Polytech), St. Petersburg, Russia, in 1994, the Ph.D. degree in electrical engineering from Lund University, Lund Sweden, in 2009, and the Docent (Habilitation) qualification in antenna systems from Chalmers University of Technology, Gothenburg, Sweden, in 2017.

From 1996-2005, he held various research and specialist positions in the Telecom industry, e.g., Ericsson Research, Telia Research, and TeliaSonera, in Stockholm, Sweden. From 2001-2005, he was the Swedish delegate to the European Cost Action 273 and from 2018-2020 he was the Dutch delegate to the European Cost Action IRACON. He has been one of the pioneers in producing the first standardized OTA measurement techniques for 3GPP, and devising novel OTA techniques, e.g., the RandomLOS and the Hybrid antenna characterization setups.

$\mathrm{He}$ has contributed to, or initiated various European research projects, e.g., more recently, the is 3DMIMO, the WAVECOMBE and the Build-Wise projects under the auspices of the H2020 European Research and Innovation program. Dr. Glazunov has also contributed to the international 3GPP and the ITU standardization bodies. From 2009-2010, he held a Marie Curie Senior Research Fellowship at the Centre for Wireless Network Design, University of Bedfordshire, Luton, U.K. From 2010-2014, he held a postdoctoral position with the Electromagnetic Engineering Laboratory, KTHRoyal Institute of Technology, Stockholm, Sweden. From 2014-2018, he held an Assistant Professor position at Chalmers University of Technology, Gothenburg, Sweden.

Dr. Glazunov is currently an Associate Professor with the Department of Electrical Engineering, University of Twente, Enschede, the Netherlands, where he is leading the Radio, Propagation and Antenna Systems research. And he is also an Affiliate Professor with the Chalmers University of Technology, Gothenburg, Sweden, where he is leading the OTA Characterization of Antenna Systems research area. He is the author of more than hundred scientific and technical publications. He is the co-author and co-editor of the text book LTE-Advanced and Next Generation Wireless Networks Channel Modelling and Propagation (Wiley, 2012). His current research interests include, but are not limited to mmWave array antenna design, MIMO antenna systems, electromagnetic theory, fundamental limitations on antennachannel interactions, radio propagation channel measurements, modeling and simulations, wireless performance in the built environment, and the OTA characterization of antenna systems and wireless devices.

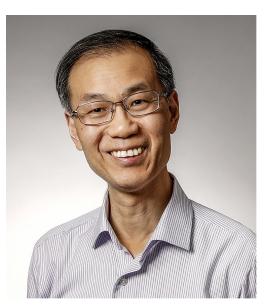

Jian Yang (M'02-SM'10) received B.S. degree from the Nanjing University of Science and Technology, Nanjing, China, in 1982, and the M.S. degree from the Nanjing Research Center of Electronic Engineering, Nanjing, China, in 1985, both in electrical engineering, and the Swedish Licentiate and Ph.D. degrees from the Chalmers University of Technology, Gothenburg, Sweden, in 1998 and 2001, respectively.

From 1985 to 1996, he was with the Nanjing Research Institute of Electronics Technology, Nanjing, China, as a Senior Engineer. From 1999 to 2005, he was with the Department of Electromagnetics, Chalmers University of Technology as a Research Engineer. During 2005 and 2006, he was with COMHAT AB as a Senior Engineer. From 2006 to 2010, he was an Assistant Professor at the Department of Signals and Systems, Chalmers University of Technology. From 2010, 2016 and 2020, he has been an Associate Professor, a Professor and a Full Professor, respectively, at the Department of Electrical Engineering, Chalmers University of Technology. He has published more than 70 journal articles and about 150 peer reviewed conference papers. H-index: 28 . His research interests include ultra-wideband antennas and UWB feeds for reflector antennas, mmWave antennas, mmWave multilayer phased array antennas, mmWave SWE (sheet waveguide element) antennas, Gap waveguide antennas, UWB radar systems, UWB antennas in near-field sensing applications, hat-fed antennas, reflector antennas, radome design, and computational electromagnetics. 